\title{
Estimação de distribuições discretas via cópulas de Bernstein
}

\author{
Victor Fossaluza \\ TESE APRESENTADA \\ AO \\ Instituto DE MATEMÁtica E EstatísticA \\ DA \\ Universidade DE SÃo PAulo \\ PARA \\ OBTENÇÃO DO TÍTULO \\ $\mathrm{DE}$ \\ Doutor EM CIÊNCIAS
}

Programa: Estatística

Orientador: Prof. Dr. Carlos Alberto de Bragança Pereira Co-orientador: Prof. Dr. Luís Gustavo Esteves

Durante o desenvolvimento deste trabalho o autor recebeu auxílio financeiro da CAPES/CNPq

São Paulo, março de 2012 


\title{
Estimação de distribuições discretas via cópulas de Bernstein
}

\author{
Esta versão definitiva da tese \\ contém as correções e alterações sugeridas pela \\ Comissão Julgadora durante a defesa realizada \\ por Victor Fossaluza em 15/03/2012.
}

Comissão Julgadora:

- Prof. Dr. Carlos Alberto de Bragança Pereira (orientador) - IME-USP

- Prof. Dr. Carlos Alberto Ribeiro Diniz - DEs-UFSCar

- Prof. Dr. Fabio Gagliardi Cozman - POLI-USP

- Profa. Dra. Helena Paula Brentani - FM-USP

- Prof. Dr. Nikolai Valtchev Kolev - IME-USP 


\section{Agradecimentos}

Em primeiro lugar, agradeço aos meus orientadores, os professores Luís Gustavo Esteves e Carlos Alberto de Bragança Pereira (o Carlinhos), que tornaram possível a realização deste trabalho. O professor Luís Gustavo acompanhou toda minha trajetória acadêmica, tendo orientado também minha iniciação científica e meu mestrado, e foi o grande responsável pelo surgimento do meu interesse pela área de fundamentos e pela inferência bayesiana. O professor Carlinhos, guru da estatística bayesiana no Brasil, esteve junto comigo desde o último ano de graduação e foi quem me incentivou a trabalhar com aplicações, ensinando desde a melhor forma de extrair informações de pesquisadores até a resolução de problemas práticos que não tinham uma solução convencional. Ao ouvir as inúmeras histórias contadas pelo professor Carlinhos sobre quanta sorte ele teve em sua carreira, pude perceber minha sorte em tê-los como mestres e amigos.

Agradeço aos meus pais, Wilma e Valdecir, primeiramente por terem me proporcionado a oportunidade de vir a este mundo. Eles são os maiores responsáveis pela minha formação como ser humano, sempre dando bons exemplos e guiando-me pelo caminho correto. Além disso, sempre deram suporte total para que eu pudesse dedicar-me aos estudos quase que integralmente. Aproveito também para agradecer aos meus irmãos, Vinícius e Vandré, que sempre estiveram por perto, me apoiaram e trouxeram alegria em momentos difíceis.

À Laís, minha namorada, que aguentou a fase mais complicada no final de meu mestrado, todo o período de doutorado, a distância (pois dei aulas na UFSCar, em São Carlos, durante o último semestre), minhas irritações e tudo mais que uma "pessoa comum" não aguentaria. Ela me deu força quando tudo parecia perdido, dando exemplos de amor e companheirismo.

A todos os meus professores e companheiros acadêmicos, que muito me ensinaram em suas aulas e conversas durante o café. Muitos nomes caberiam aqui mas, em particular, gostaria de citar os mestres Sérgio Wechsler e Júlio Stern, que sempre contribuíram com ideias e sugestões valiosas, e os amigos Patrícia Viana da Silva, Jony Arrais Pinto Junior, Cibele Maria Russo Noveli, Adriano Polpo de Campo, Marcio Alves Diniz e Lina Dornelas Thomas.

Por último, mas não menos importante, agradeço a todos os Amigos que estiveram comigo, ajudando em tudo que puderam, compartilhando experiências, dando exemplos e fortalecendo algo de inestimável valor: os princípios que regem as minhas ações. 


\section{Resumo - Estimação de Distribuições Discretas via Cópulas de Bernstein}

As relações de dependência entre variáveis aleatórias é um dos assuntos mais discutidos em probabilidade e estatística e a forma mais abrangente de estudar essas relações é por meio da distribuição conjunta. Nos últimos anos vem crescendo a utilização de cópulas para representar a estrutura de dependência entre variáveis aleatórias em uma distribuição multivariada. Contudo, ainda existe pouca literatura sobre cópulas quando as distribuições marginais são discretas. No presente trabalho será apresentada uma proposta não-paramétrica de estimação da distribuição conjunta bivariada de variáveis aleatórias discretas utilizando cópulas e polinômios de Bernstein.

Palavras-chave: Distribuições Discretas, Cópulas, Polinômios de Bernstein, Distância de Aitchison, Inferência Não Paramétrica. 


\section{Abstract - Discrete Distributions Estimation via Bernstein Copulas}

The relations of dependence between random variables is one of the most discussed topics in probability and statistics and the best way to study these relationships is through the joint distribution. In the last years has increased the use of copulas to represent the dependence structure among random variables in a multivariate distribution. However, there is still little literature on copulas when the marginal distributions are discrete. In this work we present a non-parametric approach for the estimation of the bivariate joint distribution of discrete random variables using copulas and Bernstein polynomials.

Keywords: Discrete Distributions, Copulas, Bernstein Polynomials, Aitchison Distance, Non Parametric Inference. 


\section{Sumário}

$\begin{array}{ll}\text { Lista de Abreviaturas } & \text { ix }\end{array}$

Lista de Símbolos $\quad$ xi

Lista de Figuras $\quad$ xiii

$\begin{array}{ll}\text { Lista de Tabelas } & \text { xv }\end{array}$

1 Introdução $\quad 1$

$\begin{array}{lll}2 & \text { Conceitos } & 7\end{array}$

2.1 Notação e Conceitos Básicos . . . . . . . . . . . . . . . . 7

2.2 Cópulas e Polinômios de Bernstein . . . . . . . . . . . . . . . 9

3 Estimação de Distribuições Discretas 13

3.1 Distribuição Empírica . . . . . . . . . . . . . . . . . 15

3.2 Aproximação Por Uma Distribuição Contínua - Caso Normal . . . . . . . . . 15

3.3 Aproximação por Polinômios de Bernstein . . . . . . . . . . . . . . 17

3.3.1 Aproximação Direta da Função de Distribuição . . . . . . . . . . . . 17

3.3.2 Solução Proposta: Aproximação da Função de Distribuição via Cópulas de Bernstein . . . . . . . . . . . . . . . . 18

3.4 Avaliação das Estimativas . . . . . . . . . . . . . . . . 19

4 Aplicações $\quad 23$

4.1 Definições Preliminares . . . . . . . . . . . . . . . . . . 23

4.2 Exemplo 1: Distribuição Bivariada Simétrica . . . . . . . . . . . . . . 24

4.3 Exemplo 2: Distribuição Bivariada Assimétrica . . . . . . . . . . . . . . 26

4.4 Exemplo 3: Dados de Pacientes Obsessivos-Compulsivos . . . . . . . . . . . 29

4.5 Nota sobre o grau do polinômio de Bernstein . . . . . . . . . . . . . . 31

5 Conclusões $\quad 33$

5.1 Sugestões para Pesquisas Futuras . . . . . . . . . . . . . . 36 
$\begin{array}{ll}\text { A Rotinas computacionais } & 37\end{array}$

A.1 Função de distribuição empírica univariada . . . . . . . . . . . . . . . . 37

A.2 Polinômio de Bernstein univariado . . . . . . . . . . . . . . . . 37

A.3 Função de distribuição empírica bivariada . . . . . . . . . . . . . . . . 38

A.4 Função de probabilidade empírica bivariada . . . . . . . . . . . . 38

A.5 Função de distribuição bivariada estimada pela distribuição Normal . . . . . 38

A.6 Polinômio de Bernstein bivariado . . . . . . . . . . . . . . . . . 39

A.7 Função de probabilidade estimada . . . . . . . . . . . . . . . . . 40

A.8 Distância de Aitchison . . . . . . . . . . . . . . . . . . 40

A.9 Gráficos . . . . . . . . . . . . . . . . . 40

A.10 Distribuição do Exemplo 1 . . . . . . . . . . . . . . . . . . 42

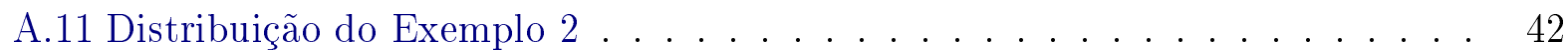

Referências Bibliográficas $\quad 45$

$\begin{array}{ll}\text { Índice Remissivo } & 49\end{array}$ 


\title{
Lista de Abreviaturas
}

\author{
v.a. Vetor aleatório. \\ f.d. Função de distribuição. \\ f.p. $\quad$ Função de probabilidade. \\ f.d.p Função de densidade de probabilidade. \\ Y-BOCS Escala de Sintomas Obsessivo Compulsivos Yale-Brown. \\ M-YBOCS Maior pontuação dentre as duas subescalas da Y-BOCS. \\ EMV Estimador de máxima verossimilhança.
}




\section{Lista de Símbolos}

$\Omega \quad$ Espaço amostral.

$\mathcal{F} \quad \sigma$-álgebra de subconjuntos de $\Omega$.

$P \quad$ Função de probabilidade.

$\mathcal{B} \quad \sigma$-álgebra de Borel.

$\boldsymbol{X} \quad$ Vetor aleatório.

F Função de distribuição de $\boldsymbol{X}$.

$\mathbb{E}[g(\boldsymbol{X})]$ Valor esperado do vetor aleatório $g(\boldsymbol{X})$, isto é, $\mathbb{E}[g(\boldsymbol{X})]=\int g(\boldsymbol{X}) d F(\boldsymbol{X})$.

$\mathbb{1}_{A}(x) \quad$ Função indicadora do conjunto $A$, isto é, $\mathbb{1}_{A}(x)=\mathbb{1}\{x \in A\}= \begin{cases}1 & \text { se } x \in A \\ 0 & \text { caso contrário. }\end{cases}$ 


\section{Lista de Figuras}

1.1 YBOCS obsessão e compulsão … . . . . . . . . . . . . . . . . 3

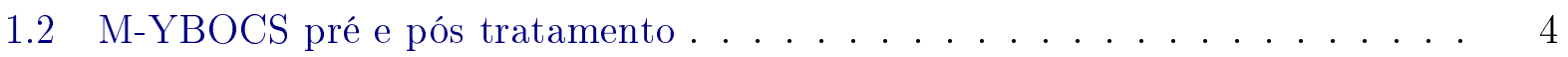

3.1 Aproximação da f.d. discreta por uma f.d. absolutamente contínua. . . . . . 14

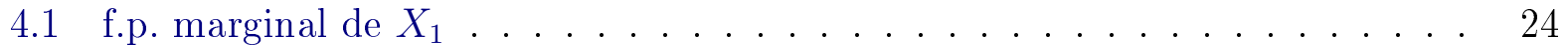

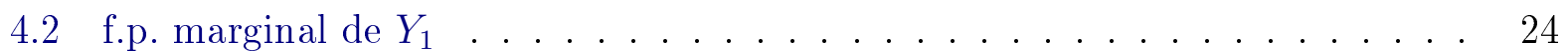

4.3 f.d. e f.p. teóricas de $\left(X_{1}, Y_{1}\right) \ldots \ldots \ldots \ldots \ldots \ldots \ldots \ldots$

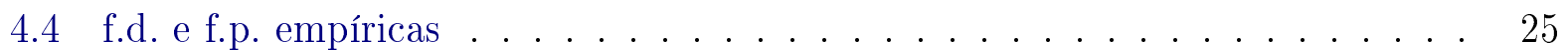

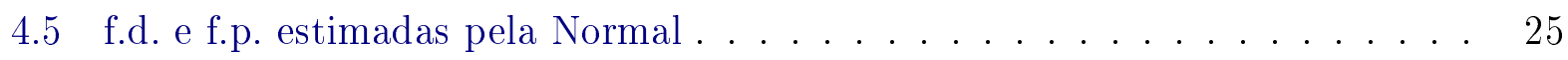

4.6 f.d. e f.p. estimadas pelo polinômio Bernstein . . . . . . . . . . . . 25

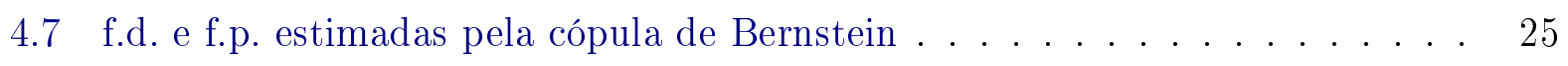

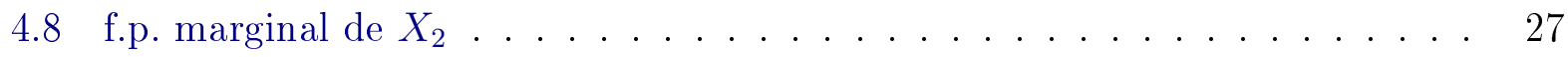

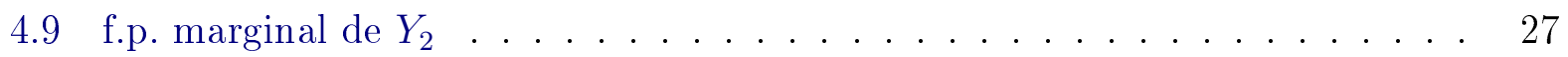

4.10 f.d. e f.p. teóricas de $\left(X_{2}, Y_{2}\right) \ldots \ldots \ldots \ldots \ldots \ldots \ldots \ldots$

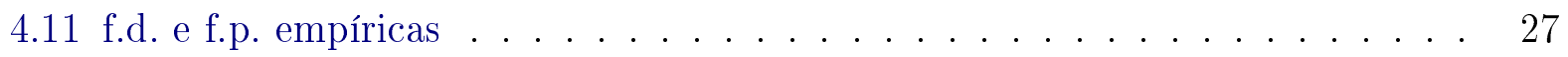

4.12 f.d. e f.p. estimadas pela Normal . . . . . . . . . . . . . . . . 27

4.13 f.d. e f.p. estimadas pelo polinômio de Bernstein . . . . . . . . . . 28

4.14 f.d. e f.p. estimadas pela cópula de Bernstein . . . . . . . . . . . 28

4.15 Frequências observadas de $X \ldots \ldots \ldots \ldots \ldots \ldots \ldots$

4.16 Frequências observadas de $Y \ldots \ldots \ldots \ldots$

4.17 Frequências observadas para o $\operatorname{par}(X, Y) \ldots \ldots \ldots \ldots \ldots \ldots$

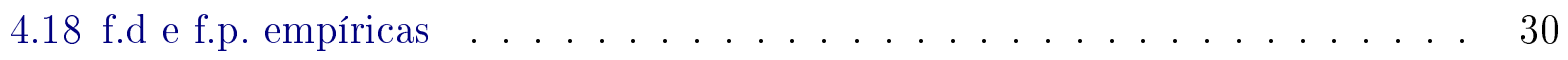

4.19 f.d. e f.p. estimadas pela Normal . . . . . . . . . . . . . . . . 30

4.20 f.d. e f.p. estimadas pelo polinômio Bernstein . . . . . . . . . . . 30

4.21 f.d e f.p. estimadas pela cópula de Bernstein . . . . . . . . . . . . 31 


\section{Lista de Tabelas}

4.1 Comparação das estimativas do exemplo da Seção 4.2 . . . . . . . . . . . 26

4.2 Comparação das estimativas do exemplo da Seção 4.3 . . . . . . . . . . . . . . . 28

4.3 Comparação das estimativas do exemplo da Seção 4.4 . . . . . . . . . . . . . 31

4.4 Comparação das estimativas para diferentes valores de $m$ no exemplo da Seção

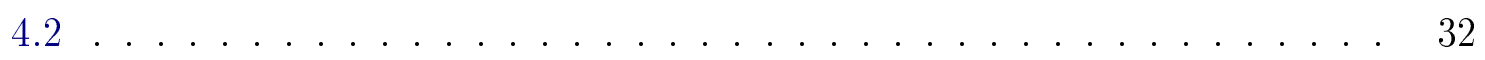

4.5 Comparação das estimativas para diferentes valores de $m$ no exemplo da Seção

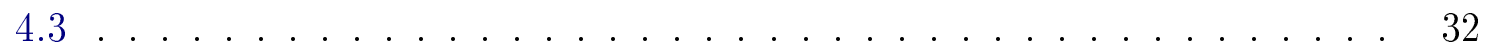


xvi LISTA DE TABELAS 


\title{
Capítulo 1
}

\section{Introdução}

\begin{abstract}
"The Statistician is the Wizard who makes 'scientific' statements about invisible states and quantities. However, contrary to the real wishes (or witches), he attaches uncertainties to his statements." (Carlos A. de B. Pereira)
\end{abstract}

Relações de dependência entre variáveis aleatórias são dos temas mais discutidos na literatura estatística e probabilística. Grande parte dos trabalhos de um estatístico envolve casos onde mais de uma variável aleatória é observada em cada uma das unidades amostrais. A relação e o nível de dependência entre essas variáveis são, na maioria das vezes, tema de interesse. No dia a dia do trabalho estatístico são raras as vezes onde não se questiona a falta ou presença de associação, forte ou fraca, entre variáveis. Por exemplo, hoje em dia é natural a pergunta se a obesidade influi na qualidade de vida de um sujeito: se $X$ é o valor do índice de massa corpórea e $Y$ é um escore obtido por um instrumento de avaliação de qualidade de vida, é natural a pergunta "Qual o nível de associação entre $X$ e $Y$ ?". Muitas são as formas de se medir associação entre variáveis e as mais comuns são o coeficiente de correlação entre $X$ e $Y$, no caso contínuo, e a estatística $\chi$-quadrado para o caso categórico. O coeficiente de correlação mede a relação linear existente entre variáveis contínuas, enquanto a estatística $\chi$-quadrado mede a associação entre as duas variáveis categóricas. Em qualquer uma das abordagens, o interesse é avaliar propriedades da distribuição conjunta do vetor aleatório $\boldsymbol{X}=(X, Y)$ (ou da distribuição condicional de $Y$ dado $X=x$, para cada valor de $x$ ).

A estrutura de dependência entre variáveis aleatórias fica definida no momento em que se estabelece o modelo estatístico que será usado. Por modelo estatístico entende-se um conjunto de famílias de distribuições de probabilidade indexadas pelo valor de um parâmetro (desconhecido) de interesse: cada valor do parâmetro define um modelo probabilístico. Quando as variáveis envolvidas são absolutamente contínuas, o modelo fica unicamente determinado por uma função de densidade de probabilidade; no caso em que as variáveis são discretas (isto é, assumem valores em um conjunto enumerável), esse modelo é caracterizado por uma função de probabilidade. Equivalentemente, o modelo pode ser determinado pela especificação de uma função de distribuição. 
Há diversos trabalhos dedicados à estimação de distribuições, em especial no caso univariado e com a variável aleatória sendo absolutamente contínua. A abordagem adotada pela maioria dos livros clássicos de estatística se restringe ao caso em que o vetor aleatório $\boldsymbol{X}$ possui distribuição pertencente à uma família de distribuições $\mathcal{P}=\left\{P_{\theta}: \boldsymbol{\theta} \in \Theta\right\}$; o conjunto de distribuições $P_{\theta}$ indexadas pelo parâmetro $\boldsymbol{\theta}$ pertencente ao espaço paramétrico $\Theta$. Assim, a estimação da função de distribuição restringe-se à estimação do parâmetro desconhecido $\boldsymbol{\theta}$. A família mais utilizada nesse tipo de abordagem é a das distribuições normais multivariadas, que tem como parâmetros o vetor de médias $\boldsymbol{\mu}$ e matriz de covariância $\boldsymbol{\Sigma}$. Recentemente, a adequabilidade da distribuição normal tem sido bastante questionada nos casos em que as caudas da distribuição são pesadas ou existe algum tipo de assimetria. Diversas alternativas tem sido apresentadas como, por exemplo, as distribuições elípticas assimétricas (veja, por exemplo, Branco e Dey (2001); Genton e Loperfido (2005)). Sob essa abordagem, a estrutura de dependência entre as variáveis restringe-se àquela suportada pela família $\mathcal{P}$. Essa suposição pode não ser razoável e outras alternativas têm sido apresentadas, como a utilização da metodologia conhecida como teoria de cópulas.

O uso de cópulas para estudar a estrutura de dependência entre variáveis aleatórias vem crescendo consideravelmente nos últimos anos. Esse objeto matemático foi apresentado inicialmente por Sklar (1959) que enunciou um teorema que prova a existência de uma função de distribuição $C$, com marginais uniformes, que relaciona a distribuição conjunta de variáveis aleatórias com suas distribuições marginais, permitindo escrever qualquer função de distribuição multivariada como função das distribuições marginais, a saber, $F\left(X_{1}, \ldots, X_{k}\right)=$ $C\left(F_{1}\left(X_{1}\right), \ldots, F_{k}\left(X_{k}\right)\right)$, onde $F$ é a função de distribuição conjunta de $\left(X_{1}, X_{2}, \ldots, X_{k}\right)$ e $F_{i}$ é a função de distribuição marginal de $X_{i}, i=1, \ldots, k$. Uma das vantagens de utilizar cópulas é a possibilidade de modelar separadamente as distribuições marginais das variáveis $X_{1}, X_{2}, \ldots, X_{k}$ e a estrutura de dependência da distribuição conjunta. As cópulas podem ser vistas também como distribuições multivariadas com marginais uniformes no intervalo $[0,1]$ e, assim, possibilitam o estudo da dependência entre variáveis aleatórias livre de escala (Fisher, 1997; Joe, 1997; Nelsen, 2006).

Uma das dificuldades encontradas na utilização de cópulas é que o teorema de Sklar garante a existência da função $C$ mas não nos diz como encontrá-la. Esse problema é bastante encontrado na literatura e existem diversas propostas de soluções. A alternativa mais utilizada é ajustar diversas famílias de cópulas e escolher uma delas usando algum critério de seleção de modelos ou algum teste de qualidade de ajuste. Outra solução é aproximar a cópula utilizando, por exemplo, a cópula empírica ou a cópula de Bernstein (Durrleman et al., 2000b). A cópula de Bernstein é obtida usando o polinômio de Bernstein para alisar a cópula empírica.

Outra dificuldade muito citada na literatura de cópulas aparece quando alguma das variáveis é discreta. O Teorema de Sklar não garante a unicidade da cópula $C$ nesse caso e, por essa razão, a estimação da cópula na presença de variáveis discretas, apesar de necessária em diversas aplicações, é um problema pouco estudado. 
Neste trabalho, o problema de estimação da função de probabilidade de variáveis discretas é motivado por uma pesquisa clínica realizada no Projeto Transtornos do Espectro Obsessivo-Compulsivo (PROTOC) do Instituto de Psiquiatria do Hospital das Clínicas da Universidade de São Paulo (IPq-HCUSP), envolvendo pacientes com transtorno obsessivocompulsivo (TOC). Nesse estudo, obteve-se dados de um instrumento de avaliação da gravidade em que o paciente com TOC se encontra, conhecido como YBOCS (Goodman et al., 1989a,b). Essa escala é composta de duas sub-escalas, uma para avaliar a gravidade das obsessões e outra para a gravidade das compulsões e cada uma varia no conjunto $\{0,1, \ldots, 20\}$. As medidas são obtidas em um instante inicial para 1001 pacientes e após um tratamento de três meses para apenas 213 desses pacientes. A Figura 1.1 representa graficamente as medidas de obsessão e compulsão dos pacientes no instante inicial do estudo. Como essas sub-escalas são altamente correlacionadas, o máximo das duas sub-escalas (M-YBOCS) será utilizado como índice de gravidade (Pereira et al., 2011). A Figura 1.2 apresenta as frequências observadas nos instantes inicial e final para os 213 pacientes. Uma pergunta que poderia ser feita nesse caso é como estimar a distribuição conjunta do M-YBOCS nos instantes inicial e final utilizando toda a informação disponível.

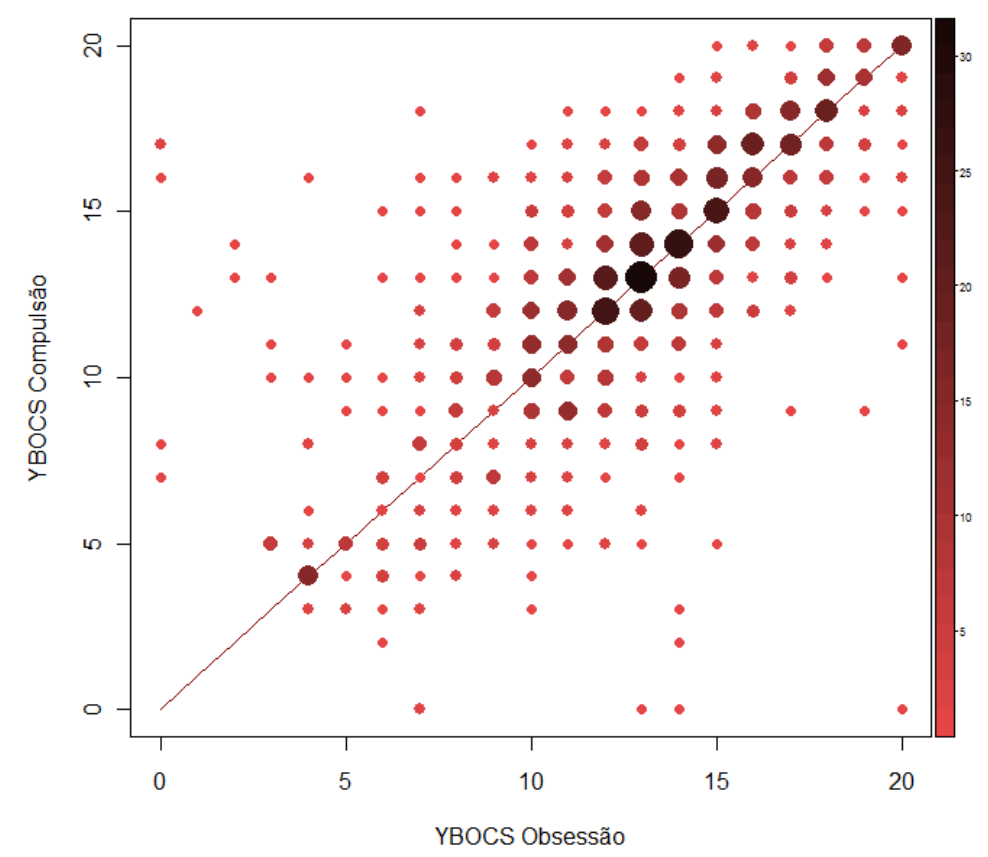

Figura 1.1: YBOCS obsessão e compulsão

A estimativa usando a frequência relativa é a primeira possível resposta. Essa estimativa, conhecida como distribuição empírica, pode ser vista como uma abordagem não paramétrica para estimar a função de distribuição. Contudo, a presença de zeros devido ao pequeno tamanho da amostra (213) comparativamente ao número de pontos a serem estimados $(21 \times 21=441)$ torna essa estimativa pobre. Outra possibilidade seria a aproximação por uma distribuição paramétrica contínua, seguida de uma discretização nos valores que a 


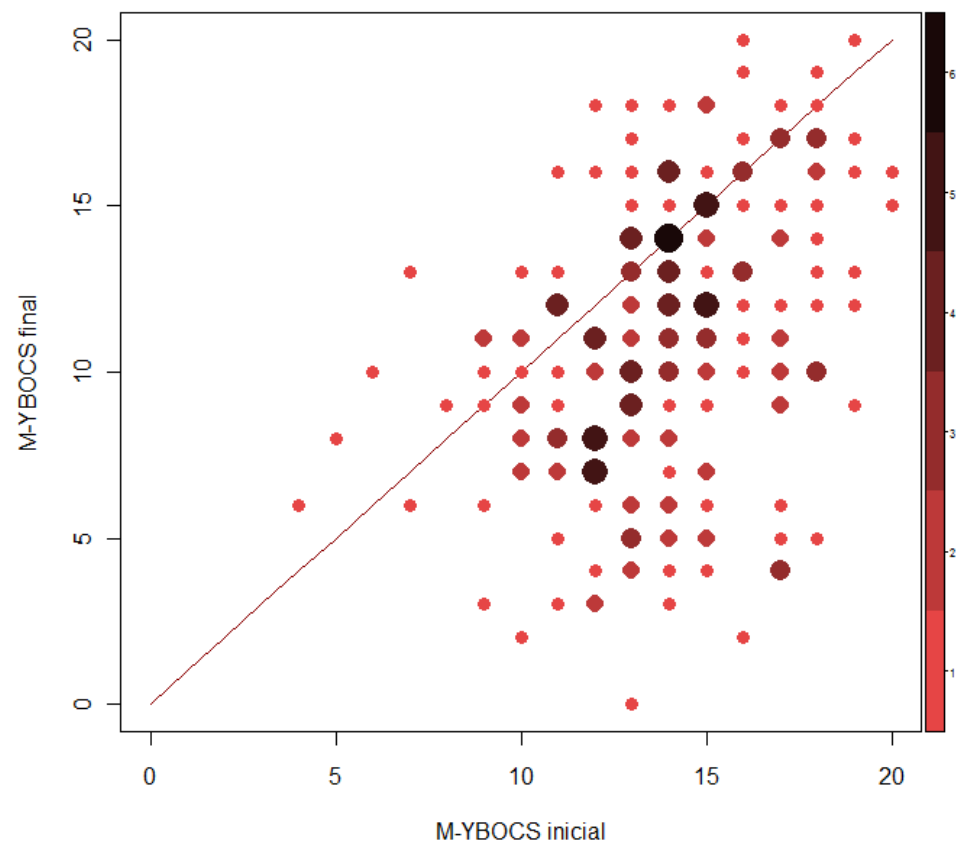

Figura 1.2: M-YBOCS pré e pós tratamento

variável, de fato, assume. Esse tipo de procedimento fica restrito à estrutura de dependência suportada por essa família de distribuições. Uma alternativa não paramétrica que permite uma estrutura de dependência mais flexível e evita o problema dos zeros é o alisamento da distribuição empírica. Essa abordagem fornece uma estimativa contínua para a função de distribuição e também torna necessária uma discretização. Contudo, essas três abordagens só permitem utilizar os 213 pares de observações.

O objetivo deste trabalho é desenvolver uma metodologia para estimar a distribuição conjunta de variáveis aleatórias discretas utilizando inclusive os dados que fornecem informação parcial, ou seja, os dados que foram observados apenas para algumas das variáveis envolvidas. O procedimento sugerido será realizado em duas etapas: a primeira consiste na estimação das distribuições marginais e, em seguida, a estimação da cópula. Essa estimação será realizada por um procedimento não paramétrico com base na função de distribuição empírica seguida de um alisamento utilizando os polinômios de Bernstein. A distribuição conjunta será então obtida utilizando a cópula para juntar as distribuições marginais. Esse procedimento será comparado com algumas outras sugestões, utilizando uma medida de distância apropriada.

As principais contribuições deste trabalho são

- introduzir a adoção dos polinômios de Bernstein para a estimação de distribuições discretas multivariadas; 
- apresentar uma metodologia para a estimação de distribuições multivariadas discretas na presença de zeros e de dados faltantes utilizando cópulas de Bernstein;

- utilizar uma medida de distância adequada para comparar as estimativas da função de probabilidade.

Ne sequência, o Capítulo 2 apresenta os conceitos básicos necessários para o desenvolvimento da tese. No Capítulo 3, quatro possíveis soluções para a estimação de distribuições discretas são propostas, além de uma medida de distância adequada para a comparação das estimativas obtidas. No Capítulo 4, esses métodos são aplicados à três conjuntos de dados, sendo que os dois primeiros foram obtidos por simulação e o último é o conjunto de dados que motivou esse trabalho, descrito anteriormente. Finalmente, no Capítulo 5, discutimos algumas conclusões desta tese e analisamos algumas das vantagens e desvantagens do método proposto.

As rotinas computacionais utilizadas neste trabalho estão disponíveis no Apêndice A. 


\section{Capítulo 2}

\section{Conceitos}

"We do not see things as they are, we see them as we are."

(Nin, 1961, p. 124)

\subsection{Notação e Conceitos Básicos}

O conjunto $\Omega$ de todos os possíveis resultados de um experimento é chamado espaço amostral. Um evento $A$ é qualquer coleção de possíveis resultados de um experimento, ou seja, um subconjunto de $\Omega(A \subseteq \Omega)$. A coleção de eventos a qual será atribuída probabilidade, denotada por $\mathcal{A}$, será uma $\sigma$-álgebra de subconjuntos de $\Omega$. A medida $\sigma$-aditiva $P^{*}: \mathcal{A} \rightarrow$ $[0,1]$, tal que $P^{*}(\Omega)=1$, é chamada medida de probabilidade. Desse modo, se $A$ é um evento de $\mathcal{A}, P^{*}(A)$ é dito a probabilidade de $A$ e será interpretado como uma medida de incerteza sobre a ocorrência do evento $A$. Dessa forma, a probabilidade atribuída a um evento pode variar de indivíduo para indivíduo; depende do conhecimento que cada um tem sobre aquele evento. A interpretação subjetivista de probabilidades ganhou força após os trabalhos de Bruno De Finetti (De Finetti, 1972, 1974) e é adotada pela escola bayesiana de inferência estatística.

Um vetor aleatório é um característico numérico dos resultados experimentais, isto é, uma função mensurável, $\boldsymbol{X}: \Omega \rightarrow \mathbb{R}^{k}, k \in \mathbb{N}$, que associa um vetor de números reais a cada possível resultado de um experimento. O conjunto $\mathcal{X}=\{\boldsymbol{X}(\omega): \omega \in \Omega\}$ é chamado espaço amostral gerado pelo vetor aleatório $\boldsymbol{X}$ e a $\sigma$-álgebra usualmente adotada nesse caso é a dos borelianos de $\mathbb{R}^{k}$, denotada por $\mathcal{F}_{k}$. Assim, a medida de probabilidade para $\boldsymbol{X}, P$, associa a cada $B \in \mathcal{F}_{k}, P(\boldsymbol{X} \in B)=P^{*}(\{\omega \in \Omega \mid X(\omega) \in B\})$. Ao trabalhar com vetores aleatórios, é suficiente definir o espaço de probabilidade $\left(\mathbb{R}^{k}, \mathcal{F}_{k}, P\right)$.

Um vetor aleatório $\boldsymbol{X}=\left(X_{1}, \ldots, X_{k}\right)$ é unicamente determinado pela sua função de distribuição (f.d.) $F(\cdot)$, definida para cada $\boldsymbol{x} \in \mathbb{R}^{k}$ como $F(\boldsymbol{x})=F\left(x_{1}, \ldots x_{k}\right)=P\left(X_{1} \leq\right.$ $\left.x_{1}, \ldots, X_{k} \leq x_{k}\right)$. Analogamente, se $\boldsymbol{X}$ é um vetor aleatório discreto, isto é, assume valores em um conjunto enumerável, ele é unicamente determinado pela sua função de probabilidade 
(f.p.) $p(\cdot)$, definida por $p(\boldsymbol{x})=P\left(X_{1}=x_{1}, \ldots, X_{k}=x_{k}\right)$, para cada $\boldsymbol{x} \in \mathcal{X}$. No caso em que $\boldsymbol{X}$ é um vetor aleatório absolutamente contínuo, ele é unicamente determinado (exceto por um conjunto de medida nula) por sua função de densidade (f.d.p.) $f(\cdot), \operatorname{com} f(\boldsymbol{x})=\frac{\partial F(\boldsymbol{x})}{\partial \boldsymbol{x}}$, $\boldsymbol{x} \in \mathbb{R}^{k}$.

Para qualquer função mensurável $g: \mathbb{R}^{k} \rightarrow \mathbb{R}^{p}, g(\boldsymbol{X})$ é também um vetor aleatório. O valor esperado do vetor aleatório $g(\boldsymbol{X})$ é $E[g(\boldsymbol{X})]=\int g(\boldsymbol{x}) d F(\boldsymbol{x})$. Em particular, o vetor de médias do vetor aleatório $\boldsymbol{X}$ é $\boldsymbol{\mu}=\left(\mu_{1}, \ldots, \mu_{k}\right)$, onde $\mu_{i}=\mathbb{E}\left[X_{i}\right], i=1, \ldots, k$. A matriz de covariância de $\boldsymbol{X}$ é dada por

$$
\boldsymbol{\Sigma}=\mathbb{E}\left[(\boldsymbol{X}-\boldsymbol{\mu})(\boldsymbol{X}-\boldsymbol{\mu})^{\prime}\right]=\left[\begin{array}{cccc}
\sigma_{1}^{2} & \sigma_{12} & \ldots & \sigma_{1 k} \\
\sigma_{12} & \sigma_{2}^{2} & \ldots & \sigma_{2 k} \\
\vdots & \vdots & \ddots & \vdots \\
\sigma_{1 k} & \sigma_{2 k} & \ldots & \sigma_{k}^{2}
\end{array}\right]
$$

onde $\sigma_{i}^{2}=\operatorname{Var}\left(X_{i}\right)$ é a variância de $X_{i}$ e $\sigma_{i j}=\operatorname{Cov}\left(X_{i}, X_{j}\right)$ é a covariância entre $X_{i}$ e $X_{j}$.

A covariância mede a força da associação linear entre duas variáveis aleatórias. Se $X$ e $Y$ são independentes então $\operatorname{Cov}(X, Y)=0$ (a recíproca não é verdadeira em geral). Grandes (pequenos) valores da covariância indicam que há uma forte associação linear positiva (negativa) entre as variáveis. Contudo, $\operatorname{como}-\infty \leq \operatorname{Cov}(X, Y) \leq \infty$ é difícil avaliar quando o valor da covariância é grande (pequeno).

Definição 2.1.1 A matriz de correlação do vetor aleatório $\boldsymbol{X}$ é dada por

$$
\boldsymbol{\rho}=\left[\begin{array}{cccc}
1 & \rho_{12} & \ldots & \rho_{1 k} \\
\rho_{12} & 1 & \ldots & \rho_{2 k} \\
\vdots & \vdots & \ddots & \vdots \\
\rho_{1 k} & \rho_{2 k} & \ldots & 1
\end{array}\right]
$$

onde $\rho_{i j}=\operatorname{cor}\left(X_{i}, X_{j}\right)=\frac{\operatorname{Cov}\left(X_{i}, X_{j}\right)}{\sqrt{\operatorname{Var}\left(X_{i}\right) \operatorname{Var}\left(X_{j}\right)}}$ é a correlação entre $X_{i}$ e $X_{j}$.

A correlação $\rho$ entre duas variáveis $X$ e $Y$ também mede a força da associação linear. Porém, diferentemente da covariância, a correlação é um número pertencente ao intervalo $[-1,1]$ e $|\rho|=1$ indica associação linear perfeita, isto é, que $Y=a X+b$ com probabilidade 1 , para $a, b \in \mathbb{R}, a \neq 0$.

Apesar de seu uso indiscriminado, o coeficiente de correlação não captura todas as formas de dependência entre variáveis aleatórias, restringindo-se à relação linear. Outras medidas de associação frequentemente usadas na literatura, como o $\tau$ de Kendall ou o $\rho$ de Spearman (Nelsen, 2006), também apresentam limitações. A forma mais abrangente de entender a associação entre variáveis aleatórias é estudar sua função de probabilidade ou de densidade conjunta. Entretanto, diferenças de escala nas variáveis também podem dificultar o entendimento da estrutura de dependência. Na próxima seção, uma abordagem alternativa - o uso de cópulas - é apresentada para o estudo da dependência entre variáveis aleatórias. 


\subsection{Cópulas e Polinômios de Bernstein}

O termo cópula deriva do verbo latino copulare que pode ser traduzido como "juntar". De forma bem geral, uma cópula pode ser definida da seguinte maneira:

Definição 2.2.1 Sejam $I=[0,1]$ e $I^{k}=[0,1] \times[0,1] \times \ldots \times[0,1]=[0,1]^{k}$. Uma $k$-cópula, $k \in \mathbb{N}$, (ou simplesmente cópula) é uma função de distribuição $C: I^{k} \rightarrow I$ de um vetor aleatório $\boldsymbol{U}=\left(U_{1}, U_{2}, \ldots, U_{k}\right)$, onde $U_{i} \sim$ Uniforme $[0,1], i=1, \ldots, k$.

Esse objeto matemático foi apresentado inicialmente por Sklar (1959) que enunciou um teorema provando a existência de uma função $C$ que relaciona a distribuição conjunta de variáveis aleatórias com suas distribuições marginais (Teorema 2.2.2).

Teorema 2.2.2 (Teorema de Sklar) Seja $F: \mathbb{R}^{k} \rightarrow[0,1]$ uma função de distribuição conjunta com marginais $F_{1}, F_{2}, \ldots, F_{k}$. Então existe uma $k$-cópula $C$ tal que, para todo $\boldsymbol{x} \in \overline{\mathbb{R}}^{k}=\mathbb{R}^{k} \cup\{-\infty, \infty\}$,

$$
F\left(x_{1}, x_{2}, \ldots, x_{k}\right)=C\left(F_{1}\left(x_{1}\right), F_{2}\left(x_{2}\right), \ldots, F_{k}\left(x_{k}\right)\right)
$$

Se $F_{1}, F_{2}, \ldots, F_{k}$ são absolutamente contínuas, $C$ é única; caso contrário, $C$ é unicamente determinada em $\operatorname{Im}\left(F_{1}\right) \times \operatorname{Im}\left(F_{2}\right) \times \ldots \times \operatorname{Im}\left(F_{k}\right)$. Reciprocamente, se $C$ é uma k-cópula e $F_{1}, F_{2}, \ldots, F_{k}$ são funções de distribuição, então $F$ definida pela expressão (2.1) é uma função de distribuição com marginais $F_{1}, F_{2}, \ldots, F_{k}$.

As cópulas permitem modelar separadamente as distribuições marginais e a estrutura de dependência entre variáveis aleatórias. Por esse motivo, o interesse pela utilização de cópulas vem crescendo consideravelmente nos últimos anos. Além disso, também podem ser vistas como distribuições multivariadas com marginais uniformes definidas no intervalo $[0,1]$ e, assim, possibilitam o estudo da dependência entre variáveis aleatórias livre de escala (Fisher, 1997; Joe, 1997; Nelsen, 2006).

Alguns exemplos de cópulas serão apresentados a seguir. Como o foco deste trabalho são distribuições bivariadas, serão exibidos exemplos de cópulas bidimensionais mas que podem ser estendidos para o caso $k$-dimensional (Nelsen, 2006).

Exemplo 2.2.3 Sejam $X$ e $Y$ variáveis aleatórias contínuas com distribuição conjunta $H$ e marginais $F$ e $G$, respectivamente. Então, a função $C: I^{2} \rightarrow I$ dada por

$$
C(u, v)=H\left(F^{-1}(u), G^{-1}(v)\right)
$$

é uma cópula. Esse modo de definir $C$ constitui um método para construir cópulas baseado em funções de distribuição conjuntas. 
Exemplo 2.2.4 (Cópula produto) Sejam $X$ e $Y$ variáveis aleatórias contínuas. $X$ e $Y$ são independentes se, e somente se, a cópula correspondente à distribuição conjunta $F$ é $C(u, v)=\Pi(u, v)=u v$. Nesse caso, $\Pi$ é chamada cópula produto.

Exemplo 2.2.5 (Limites de Fréchet-Hoeffding) Seja $C$ uma cópula. Então, para todo $(u, v) \in I^{2}, \max (u+v-1,0) \leq C(u, v) \leq \min (u, v)$. As funções $W(u, v)=\max (u+v-1,0)$ e $M(u, v)=\min (u, v)$ também são cópulas.

Exemplo 2.2.6 (Cópulas Archimedianas) Seja $\varphi: I \rightarrow[0, \infty]$ uma função contínua, convexa e estritamente decrescente tal que $\varphi(1)=0$. Considere também a pseudo-inversa de $\varphi$, $\varphi^{[-1]}:[0, \infty] \rightarrow I$, dada por $\varphi^{[-1]}(t)=\varphi^{-1}(t) \mathbb{1}\{0 \leq t \leq \varphi(0)\}$. Então, a função $C: I^{2} \rightarrow I$ dada por

$$
C(u, v)=\varphi^{[-1]}(\varphi(u)+\varphi(v))
$$

é chamada cópula archimediana e a função $\varphi$ é chamada função geradora da cópula $C$.

Na prática, as cópulas archimedianas são muito utilizadas para modelar diferentes estruturas de dependência. Outros exemplos de cópulas podem ser encontrados em Nelsen (2006).

Uma das dificuldades da utilização de cópulas ocorre no caso em que alguma das variáveis envolvidas é discreta. Se $X$ é uma variável aleatória absolutamente contínua com função de distribuição $F$, a variável aleatória $F(X)$ tem distribuição uniforme no intervalo $[0,1]$. A seguir, segue uma demonstração bastante elegante desse fato apresentada pelo prof. Dr. Debrabata Basu, em correspondência destinada ao prof. Dr. Carlos Alberto de Bragança Pereira, com o título "A Good Proof is a Master Key".

"In a hotel there are many rooms each with its individual key. Each key is made so crooked that it can open only one door lock. Only the hotel manager has the master key that is simple enough to turn inside all the locks. A good proof is such a master key. It is simple and straightforward and can unlock many a mathematical problem. Here is an example.

Let $X$ be a real random variable with c.d.f. $F$. When and how does it follow that $F(X) \sim$ $U$ (the uniform distribution over the unit interval)? It is my experience that, when this simple question is posed before a class of advanced graduate students, I get a surprising variety of crooked solutions. The master key to this problem is

(A) Whatever the c.d.f. $F$, the two events (i) $X \leq x$ and (ii) $F(X) \leq F(x)$ are identical for any fixed $x$.

Since $F$ is monotone non-decreasing, (i) implies (ii) logically. In order to understand the reverse implication, consider the set $\mathbb{I}_{x}=\{t: F(t)=F(x)\}$. Since $F$ is non-decreasing, $\mathbb{I}_{x}$ 
has to be an interval containing $x$. [If $F$ is strictly increasing at $x$, then $\mathbb{I}_{x}$ is the single-point set $\{x\}$ and in this case clearly (ii) implies (i).] Since $F$ is everywhere right-continuous, the interval $\mathbb{I}_{x}$ is always closed at its left end. The right end-point $b_{x}$ belongs to $\mathbb{I}_{x}$ if and only if $F$ is continuous at $b_{x}$. If $b_{x} \in \mathbb{I}_{x}$ then (ii) logically implies the event $X \leq b_{x}$ and this in turn implies the event $X \leq x$ since the interval $\left(x, b_{x}\right]$ has $F$-measure zero. On the other hand, if $b_{x} \notin \mathbb{I}_{x}$ then we reach the same conclusion after replacing $X \leq b_{x}$ by $X<b_{x}$ and $\left(x, b_{x}\right]$ by $\left(x, b_{x}\right)$ in the above argument. Thus $(\mathrm{A})$ is always true. It imediately follows that

(B) $P[F(X) \leq F(x)]=F(x)$ for all $x$.

If $F$ is continuous, then $F(x)$ takes all values in the interval $(0,1)$ and so $F(X) \sim U$.

$I$ have found many students struggling hard through text-book proofs of Kolmogorov's famous result that with $n$ i.i.d. random variables $X_{1}, X_{2}, \ldots, X_{n}$ with common continuous c.d.f. F, the quantity

$$
D=\sup \left|F_{n}(x)-F(x)\right|
$$

(where $F_{n}(x)$ is the empirical c.d.f.) is distribution-free. A quick turn of the master key (A) easily unlocks the problem. Writing $\mathbb{1}(E)$ for the indicator ofthe event $E$, we can rewrite $D$ as

$$
\begin{aligned}
D=\sup _{x} \mid \frac{1}{n} \sum \mathbb{I}\left(X_{j}\right. & \leq x)-F(x)\left|=\sup _{x}\right| \frac{1}{n} \sum \mathbb{I}\left(F\left(X_{j}\right) \leq F(x)\right)-F(x) \mid \\
& =\sup _{y}\left|\frac{1}{n} \sum \mathbb{I}\left(Y_{j} \leq y\right)-y\right|
\end{aligned}
$$

where $y \in(0,1)$ and $Y_{1}, Y_{2}, \ldots, Y_{n}$ are i.i.d. $U$.

Making a right-left inversion of (B) we have

(C) If $T$ is a real random variable with $G(t)=P(T \geq t)$, then $P[G(T) \leq G(t)]=G(t)$ for all $t$.

For a crooked version of the above simple master key refer to theorem (9.1) on pag 224 of Kempthorne e Folks (1971) - the authors call this the fundamental theorem of test of significance. The context is as follows. Let $H_{0}$ be a simples null-hypothesis to be tested and let $T=T(X)$ be the test criterion - the larger the observed value of $T$ the more significant is the test. For an observed value $t$ of $T$, let us call $G(t)=P\left(T \geq t \mid H_{0}\right)$ the attained level of significance. R. A. Fisher noted that if we consistently follow (in repeated trials) the rule 'reject the null hypothesis if the attained level of significance is $\leq \alpha_{0}$ ', then the probability of first kind of error associated wih this rule is $\alpha_{0}$. My advanced graduate students invariably get greatly puzzled when I ask them to prove Fisher's statement. Is the statement always true? The answer is yes provided $\alpha_{0}$ is an attainable level of significance. Thus $(\mathrm{C})$ is the so-called fundamental theorem of test of significance." 
Se $X$ é uma variável discreta, a variável $F(X)$ não tem distribuição uniforme no intervalo $(0,1)$. Além disso, a função cópula $C$ descrita no Teorema 2.2.2 não é única quando alguma das variáveis envolvidas é discreta.

Outra dificuldade aparece pois o teorema de Sklar garante a existência da cópula $C$ mas não nos diz como encontrá-la. Esse problema é bastante discutido na literatura e existem diversas propostas de soluções (veja, por exemplo, Durrleman et al. (2000b)). A mais utilizada consiste em ajustar diversas famílias (paramétricas) de cópulas e escolher uma delas usando algum critério de seleção de modelos ou algum teste de qualidade de ajuste (Berg, 2009; Genest et al., 2009). Outra solução é usar aproximações para a cópula, como a cópula empírica ou a cópula de Bernstein. A cópula de Bernstein é obtida pela aproximação de uma cópula $C$ utilizando o polinômio de Bernstein (definido a seguir).

Definição 2.2.1 Seja $h: I^{k} \rightarrow \mathbb{R}$ uma função contínua. O polinômio de Bernstein de ordem $m$ para a função $h$ é dado por

$$
B_{h}^{m}\left(x_{1}, \ldots, x_{k}\right)=\sum_{j_{1}=0}^{m} \ldots \sum_{j_{k}=0}^{m} h\left(\frac{j_{1}}{m}, \ldots, \frac{j_{k}}{m}\right) \prod_{i=1}^{k}\left(\begin{array}{c}
m \\
j_{i}
\end{array}\right) x_{i}^{j_{i}}\left(1-x_{i}\right)^{m-j_{i}}
$$

O polinômio de Bernstein é bem geral e pode ser usado para aproximar qualquer função real contínua definida em $I^{k}$. Se a função a ser aproximada tiver um domínio diferente de $I^{k}$, é possível usar uma transformação de mudança de escala antes da aproximação (veja, por exemplo, Babu et al. (2002)). Lorentz (1986) apresenta um estudo bastante detalhado sobre o polinômio de Bernstein no caso $k=1$. O teorema de Weierstrass (DeVore e Lorentz, 1993) garante o resultado enunciado a seguir (uma demonstração probabilística desse teorema, utilizando polinômios de Bernstein, pode ser encontrada em Koralov e Sinai (2007)).

Teorema 2.2.2 Seja $C: I^{k} \rightarrow \mathbb{R}$ uma função contínua. A sequência de polinômios de Bernstein $B_{C}^{m}$ converge uniformemente para a função $C$ quando $m \uparrow \infty$.

Como visto anteriormente, uma $k$-cópula é uma função $C: I^{k} \rightarrow I$ e o polinômio de Bernstein parece ser uma aproximação natural para $C$.

Definição 2.2.3 Seja $C: I^{k} \rightarrow I$ uma k-cópula e $B_{h}^{m}$ o polinômio de Bernstein de grau $m$ para aproximar uma função $h: I^{k} \rightarrow \mathbb{R}$. A aproximação $B_{C}^{m}$ para a k-cópula $C$ é chamada cópula de Bernstein e também é uma k-cópula (Sancetta e Satchell, 2004b).

Li et al. (1997, 1998), Kulpa (1999), Durrleman et al. (2000a,b), Sancetta (2004), Sancetta e Satchell (2004a,b), Taylor (2009), Bouezmarni et al. (2010) apresentaram resultados de convergência, propriedades assintóticas e aplicações das cópulas de Bernstein. 


\section{Capítulo 3}

\section{Estimação de Distribuições Discretas}

"All exact science is dominated by the idea of approximation."

(Bertrand Russell)

A estimação de distribuições discretas multivariadas é um assunto pouco discutido na literatura estatística. O caso mais estudado ocorre quando se supõe que os dados são gerados por um modelo $\mathcal{P}_{\theta}$, isto é, um conjunto de distribuições com a mesma forma funcional e indexadas por um parâmetro (desconhecido) $\theta$. Nesse caso, a estimação do parâmetro $\theta$ é comumente realizada utilizando métodos de máxima verossimilhança ou estimadores de Bayes, como a média ou a moda da distribuição posterior para $\theta$. Entretanto, fixar um modelo paramétrico nem sempre é razoável e pode gerar estimativas que não são satisfatórias. Na Seção 3.2, são apresentados exemplos usando essa abordagem para o caso da distribuição normal.

A estimação não paramétrica para distribuições discretas, em geral, limita-se às frequências relativas. Amostras obtidas de um vetor aleatório $\boldsymbol{X}$ podem apresentar frequências iguais a zero para um ou mais valores possíveis de $\boldsymbol{X}$. Em particular, isso ocorre quando a razão $n / d$ é menor do que 1 , onde $n$ é o tamanho da amostra e $d$ é o número de pontos que compõem o suporte da distribuição de $\boldsymbol{X}$ (que, nesse caso, coincide com a dimensão do espaço paramétrico). Uma possível solução para esse problema é aproximar a distribuição discreta (desconhecida) por uma distribuição absolutamente contínua $\hat{F}$ e, em seguida, "discretizá-la". Para isso, basta notar que se $X$ é uma variável aleatória discreta com função de distribuição $F$ assumindo valores no conjunto $\left\{a_{1}, a_{2}, a_{3}, \ldots\right\}$ com probabilidade 1 , com $a_{i}<a_{i+1}$, então $p\left(a_{i}\right)=P\left(X=a_{i}\right)=\Delta^{1}\left(a_{i}, a_{i-1}\right)=F\left(a_{i}\right)-F\left(a_{i-1}\right), i \in\{1,2, \ldots\}$ e $a_{0} \leq a_{1}$ tal que $F\left(a_{0}\right)=0$. A figura 3.1 ilustra a aproximação por uma distribuição contínua no caso univariado. 


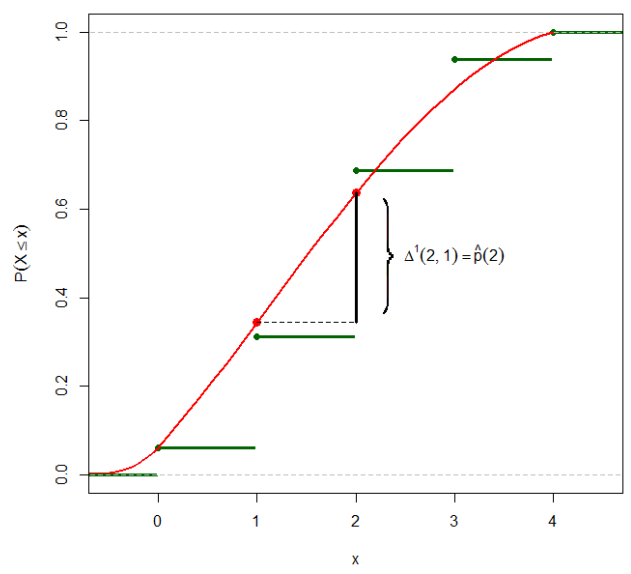

Figura 3.1: Aproximação da f.d. discreta por uma f.d. absolutamente contínua.

No caso bivariado, considere que o vetor aleatório $(X, Y)$ assume valores em $\mathcal{X}_{1} \times \mathcal{X}_{2}$, onde $\mathcal{X}_{1}=\left\{a_{1}, a_{2}, a_{3}, \ldots\right\}$ e $\mathcal{X}_{2}=\left\{b_{1}, b_{2}, b_{3}, \ldots\right\}$, respectivamente. Então

$$
\begin{gathered}
p\left(a_{i}, b_{j}\right)=P\left[(X, Y)=\left(a_{i}, b_{j}\right)\right]=\Delta^{2}\left(\left(a_{i}, b_{j}\right),\left(a_{i-1}, b_{j-1}\right)\right)= \\
=F\left(a_{i}, b_{j}\right)-F\left(a_{i-1}, b_{j}\right)-F\left(a_{i}, b_{j-1}\right)+F\left(a_{i-1}, b_{j-1}\right),
\end{gathered}
$$

com $a_{i} \leq a_{i+1}, b_{j} \leq b_{j+1}, i, j \in\{1,2,3 \ldots\}$ e $a_{0}<a_{1}$ e $b_{0}<b_{1}$ tal que $F\left(a_{0}, t\right)=F\left(t, b_{0}\right)=0$, $\forall t \in \mathbb{R}$. Usando a função $\Delta^{2}$ também é possível calcular outras probabilidades. Por exemplo,

$$
\begin{gathered}
P\left(x_{i}<X \leq x_{l}, y_{j}<Y \leq y_{m}\right)=\Delta^{2}\left(\left(x_{l}, y_{m}\right),\left(x_{i}, y_{j}\right)\right)= \\
=F\left(x_{l}, y_{m}\right)-F\left(x_{i}, y_{m}\right)-F\left(x_{l}, y_{j}\right)+F\left(x_{i}, y_{j}\right),
\end{gathered}
$$

$\operatorname{com} x_{i}, x_{l} \in \mathcal{X}_{1}, y_{j}, y_{m} \in \mathcal{X}_{2}, x_{i} \leq x_{l}, y_{j} \leq y_{m}$ e $F\left(a_{0}, t\right)=F\left(t, b_{0}\right)=0, \forall t \in \mathbb{R}$. A função $\Delta^{k}$ pode ser facilmente estendida para dimensões maiores $(k>2)$ (veja, por exemplo, Nelsen (2006)).

Nas próximas seções são apresentadas algumas sugestões para estimar uma distribuição $k$-variada discreta $F$. Considere uma amostra de tamanho $n$ de vetores aleatórios $\boldsymbol{X}_{1}, \ldots, \boldsymbol{X}_{n}$ que, condicional ao conhecimento de $F$, são independentes e identicamente distribuídos segundo $F, \operatorname{com} \boldsymbol{X}_{j}=\left(X_{1 j}, \ldots, X_{k j}\right), j=1, \ldots, n$. Após observada a amostra, os vetores aleatórios $\boldsymbol{X}_{1}, \boldsymbol{X}_{2}, \ldots, \boldsymbol{X}_{n}$ assumem os valores $\boldsymbol{x}_{1}, \boldsymbol{x}_{2}, \ldots, \boldsymbol{x}_{n}, \operatorname{com} \boldsymbol{x}_{j}=\left(x_{1 j}, \ldots, x_{k j}\right), j=$ $1, \ldots, n$. São apresentados alguns exemplos em que é considerado o caso bivariado $(k=2)$. O objetivo é estimar a função de distribuição $F \in \mathcal{D}$, onde $\mathcal{D}$ é o conjunto das funções de distribuição discretas bivariadas com o mesmo suporte de $F$. 


\subsection{Distribuição Empírica}

Definição 3.1.1 Seja $\boldsymbol{x}_{1}, \boldsymbol{x}_{2}, \ldots, \boldsymbol{x}_{n}$, com $\boldsymbol{x}_{i}=\left(x_{1 i}, \ldots, x_{k i}\right)$, uma amostra de tamanho $n$ do vetor aleatório $\boldsymbol{X}$. A função de distribuição empírica $\tilde{F}_{n}: \mathbb{R}^{k} \rightarrow[0,1]$ é definida, para cada $\boldsymbol{t}=\left(t_{1}, \ldots, t_{k}\right) \in \mathbb{R}^{k}$, como

$$
\tilde{F}_{n}(\boldsymbol{t})=\frac{1}{n} \sum_{i=1}^{n} \mathbb{1}\left\{x_{1 i} \leq t_{1}, \ldots, x_{k i} \leq t_{k}\right\}
$$

Teorema 3.1.2 (Teorema de Glivenko-Cantelli) A função de distribuição empírica $\tilde{F}_{n}$ converge uniformemente para a distribuição $F$, isto é,

$$
\tilde{F}_{n} \longrightarrow F, \text { quando } n \uparrow \infty \text { (Sen et al., 2010). }
$$

A função de distribuição empírica é muito utilizada, direta ou indiretamente, em grande parte dos métodos de inferência não paramétrica. No caso particular onde o vetor $\boldsymbol{X}$ é discreto, sua utilização é equivalente ao uso das frequências relativas para estimar a função de probabilidade, ou seja, $\tilde{p}_{n}(\boldsymbol{t})=\frac{1}{n} \sum_{i=1}^{n} \mathbb{1}\left\{\boldsymbol{x}_{i}=\boldsymbol{t}\right\}$.

Quando o tamanho da amostra $n$ é muito maior do que o número de pontos que compõem o suporte da distribuição de $\boldsymbol{X}$, esse método apresenta boas estimativas. O grande problema de sua utilização para estimar a função de distribuição conjunta $F$ (ou, equivalentemente, a função de probabilidade $p$ ) é que as probabilidades dos pontos que não foram observados na amostra são estimadas como zero.

\subsection{Aproximação Por Uma Distribuição Contínua - Caso Normal}

Uma possível solução para contornar o problema das probabilidades estimadas como zero, apresentado na seção anterior, é utilizar uma aproximação por uma distribuição contínua. Devido às suas boas propriedades matemáticas, a distribuição normal é, provavelmente, a mais utilizada na estatística e será adotada aqui como exemplo.

Definição 3.2.1 O vetor $\boldsymbol{Y}$ tem distribuição normal multivariada com vetor de médias $\boldsymbol{\mu}$ e matriz de covariância $\boldsymbol{\Sigma}\left(\boldsymbol{Y} \sim N_{k}(\boldsymbol{\mu}, \boldsymbol{\Sigma})\right)$ se sua função de densidade de probabilidade é dada por

$$
f(\boldsymbol{y})=\left[(2 \pi)^{k}|\boldsymbol{\Sigma}|\right]^{-1 / 2} \exp \left\{-\frac{1}{2}(\boldsymbol{y}-\boldsymbol{\mu})^{\prime} \boldsymbol{\Sigma}^{-1}(\boldsymbol{y}-\boldsymbol{\mu})\right\} .
$$

A função de distribuição de $\boldsymbol{Y}$ é dada por

$$
F(\boldsymbol{y})=\int_{-\infty}^{y_{1}} \ldots \int_{-\infty}^{y_{k}} f(\boldsymbol{y}) d y_{k} \ldots d y_{1} .
$$


No caso em que $\boldsymbol{\mu}=\mathbf{0}$ e $\boldsymbol{\Sigma}=\boldsymbol{I}$, onde $\mathbf{0}$ é um vetor de zeros e $\boldsymbol{I}$ é a matriz identidade, diz-se que $\boldsymbol{Y}$ tem distribuição normal padrão k-variada e a função de distribuição é denotada por $\Phi$.

A estimação dos parâmetros pode ser feita de diversas maneiras. O método mais usado na literatura estatística é a estimação por máxima verossimilhança e será o adotado neste caso. O estimador de máxima verossimilhança (EMV) também apresenta boas propriedades matemáticas e pode ser interpretado como o valor dos parâmetros que torna a amostra observada a mais provável. Além disso, esse estimador coincide com o estimador bayesiano quando a função perda 0 -1 é considerada e a distribuição imprópria $f(\boldsymbol{\mu}, \boldsymbol{\Sigma}) \propto 1$ é adotada como priori para $(\boldsymbol{\mu}, \boldsymbol{\Sigma})$ (Paulino et al., 2003).

Resultado 3.2.2 Seja $\boldsymbol{Y}_{1}, \ldots, \boldsymbol{Y}_{n}$ uma amostra aleatória de uma população normal multivariada com parâmetros $\boldsymbol{\mu}$ e $\boldsymbol{\Sigma}$. A função de verossimilhança é dada por

$$
L\left(\boldsymbol{\mu}, \boldsymbol{\Sigma} \mid \boldsymbol{y}_{1}, \ldots, \boldsymbol{y}_{n}\right)=\left[(2 \pi)^{k}|\boldsymbol{\Sigma}|\right]^{-n / 2} \exp \left\{-\frac{1}{2} \sum_{i=1}^{n}\left(\boldsymbol{y}_{i}-\boldsymbol{\mu}\right)^{\prime} \boldsymbol{\Sigma}^{-1}\left(\boldsymbol{y}_{i}-\boldsymbol{\mu}\right)\right\}
$$

Neste caso, os estimadores de máxima verossimilhança são

$$
\hat{\boldsymbol{\mu}}=\overline{\boldsymbol{Y}} \quad e \quad \hat{\boldsymbol{\Sigma}}=\frac{1}{n} \sum_{i=1}^{n}\left(\boldsymbol{Y}_{i}-\overline{\boldsymbol{Y}}\right)\left(\boldsymbol{Y}_{i}-\overline{\boldsymbol{Y}}\right)^{\prime}
$$

No caso $k=2$ o domínio da distribuição normal é $\mathbb{R}^{2}$ e para um vetor aleatório $\boldsymbol{X}=(X, Y)$ que varia no conjunto $\mathcal{X}_{1} \times \mathcal{X}_{2}, \operatorname{com} \mathcal{X}_{i}=\left\{a_{i}=a_{i 1}, \ldots, a_{i r_{i}}=b_{i}\right\}, i=1,2$, será necessário truncar a normal no conjunto $\left[a_{1}, b_{1}\right] \times\left[a_{2}, b_{2}\right]$. A função de probabilidade de $\boldsymbol{X}$ pode então ser estimada usando a função $\Delta^{2}$ (página 13 ).

As estimativas pela distribuição normal podem ser melhores em relação àquelas obtidas usando a distribuição empírica pois não atribui zero às probabilidades dos valores não observados. Como será exibido nos exemplos do Capítulo 4, essas estimativas melhoram quando a distribuição a ser estimada é aproximadamente simétrica. Nos problemas em que a distribuição a ser estimada for assimétrica, a aproximação normal pode não ser razoável. Além disso, nos casos multivariados, a distribuição normal não permite modelar diferentes estruturas de dependência. Nesse caso, outras famílias de distribuições (paramétricas) poderiam ser testadas, mas esse não é o objetivo deste trabalho e vamos nos restringir ao exemplo da normal. Na Seção 3.3 são apresentadas duas soluções alternativas que não apresentam as limitações descritas para a distribuição normal. 


\subsection{Aproximação por Polinômios de Bernstein}

O polinômio de Bernstein, apresentado no capítulo anterior, pode ser usado para aproximar funções de densidade ou de distribuição contínuas. Os polinômios de Bernstein já foram utilizados no caso univariado para aproximar funções de distribuição e densidade de variáveis aleatórias contínuas (veja, por exemplo, Petrone (1999a,b); Ghosal (2001); Petrone e Wasserman (2002) para uma abordagem bayesiana para esse problema e Babu et al. (2002) para uma abordagem frequentista). Perez e Palacín (1987); Cheng (1995); Cheng e Parzen (1997) utilizaram polinômios de Bernstein para estimar a função quantílica $F^{-1}$ de variáveis aleatórias. Quando o domínio da variável $X$ é um intervalo compacto $[a, b], a<b$, uma transformação do tipo $Y=(X-a) /(b-a)$ permite a utilização do polinômio de Bernstein para aproximar a função de distribuição de $X$. Babu et al. (2002) sugerem transformações para variáveis que não são definidas num intervalo compacto. Por exemplo, as transformações $Y=X /(1+X)$ e $Y=(1 / 2)+(1 / \pi) \tan ^{-1}(X)$ podem ser usadas nos casos em que a variável $X$ tem suporte $[0, \infty)$ ou $(-\infty, \infty)$, respectivamente. Babu e Chaubey (2006) utilizam os polinômios de Bernstein para a estimação de funções de distribuição contínuas também no caso multivariado.

\subsubsection{Aproximação Direta da Função de Distribuição}

A primeira solução dada aqui será usar polinômios de Bernstein para aproximar a função distribuição $F$ do vetor aleatório discreto $\boldsymbol{X}=(X, Y)$. Como a função $F$ é desconhecida, será utilizado o polinômio de Bernstein $B_{\tilde{F}_{m}}^{m}$ para alisar a distribuição empírica $\tilde{F}_{n}$ (Babu e Chaubey (2006)). Além disso, como o vetor aleatório $\boldsymbol{X}=(X, Y)$ varia no conjunto $\mathcal{X}_{1} \times \mathcal{X}_{2}$, com $\mathcal{X}_{i}=\left\{a_{i}=a_{i 1}, \ldots, a_{i r_{i}}=b_{i}\right\}, i=1,2$, serão utilizadas as transformações $Y_{i}=\left(X_{i}-a_{i}\right) /\left(b_{i}-a_{i}\right), i=1,2$, de modo que o domínio de $Y_{i}$ será um conjunto de valores no intervalo $[0,1]$. A função de distribuição estimada será dada por:

$$
\hat{F}_{B}\left(y_{1}, y_{2}\right)=B_{\tilde{F}_{n}}^{m}\left(y_{1}, y_{2}\right)=\sum_{j_{1}=0}^{m} \sum_{j_{2}=0}^{m} \tilde{F}_{n}\left(\frac{j_{1}}{m}, \frac{j_{2}}{m}\right) \prod_{i=1}^{2}\left(\begin{array}{c}
m \\
j_{i}
\end{array}\right) y_{i}{ }^{j_{i}}\left(1-y_{i}\right)^{m-j_{i}}
$$

onde $\tilde{F}_{n}$ é a função de distribuição empírica definida na Seção 3.1.

Após a função de distribuição ser estimada dessa forma, a função de probabilidade será obtida novamente usando a função $\Delta^{2}$ (página 13).

Diferentemente da estimação usando uma distribuição paramétrica, como àquela apresentada na seção anterior, essa solução não restringe a estrutura de dependência da distribuição conjunta, permitindo modelar diferentes formas de associação entre as variáveis. Contudo, assim como as soluções apresentadas anteriormente, somente são utilizadas as observações completas. A solução proposta a seguir permite utilizar toda a informação contida na amostra, inclusive os dados que foram observadas apenas para uma das variáveis. 


\subsubsection{Solução Proposta: Aproximação da Função de Distribuição via Cópulas de Bernstein}

No exemplo que motivou este trabalho, apresentado na introdução, há observações que foram feitas apenas para uma das variáveis e, nas soluções apresentadas anteriormente, a estimação da distribuição conjunta é realizada utilizando apenas as observações completas, isto é, somente os dados que foram observadas apenas todas as variáveis. A solução que será proposta aqui pretende utilizar toda a informação contida na amostra, inclusive nos casos onde há dados perdidos para alguma das variáveis. Para isso, as distribuições marginais serão estimadas utilizando todas as observações disponíveis para cada uma das variáveis, a cópula será estimada usando as observações completas e a estimativa para a distribuição conjunta será baseada no Teorema de Sklar (Teorema 2.2.2), utilizando as estimativas para as distribuições marginais e a cópula.

Para tal, a aproximação da função de distribuição marginal $F_{i}$ da variável $X_{i}$ será feita utilizando uma versão univariada do procedimento descrito na seção anterior, com base na função de distribuição empírica e no polinômio de Bernstein. A estimação da cópula também será feita como na seção anterior, porém utilizando a cópula empírica, definida a seguir (Nelsen (2006)).

Definição 3.3.1 Seja $\boldsymbol{x}_{1}, \boldsymbol{x}_{2}, \ldots, \boldsymbol{x}_{n}, \operatorname{com} \boldsymbol{x}_{i}=\left(x_{i}, y_{i}\right), i=1, \ldots$, uma amostra de tamanho $n$ do vetor aleatório $\boldsymbol{X}=(X, Y)$. A cópula empírica é a função $\tilde{C}_{n}$ definida por

$$
\tilde{C}_{n}\left(\frac{i}{n}, \frac{j}{n}\right)=\frac{1}{n} \sum_{k=1}^{n} \mathbb{1}\left\{x_{k} \leq x_{(i)}, y_{k} \leq y_{(j)}\right\}
$$

onde $x_{(i)}$ e $y_{(j)}, 1 \leq i, j \leq n$ denotam as estatísticas de ordem para as observações de $X e$ $Y$, respectivamente.

Caso as distribuições marginais $F_{1}$ e $F_{2}$ de $\boldsymbol{X}$ fossem conhecidas, as observações "verdadeiras" da cópula correspondente à amostra $\boldsymbol{X}_{1}, \ldots, \boldsymbol{X}_{n} \operatorname{seriam} \boldsymbol{U}_{1}^{*}, \ldots, \boldsymbol{U}_{n}^{*}, \operatorname{com} \boldsymbol{U}_{i}^{*}=$ $\left(F_{1}\left(X_{i}\right), F_{2}\left(Y_{i}\right)\right)$. Considere as funções de distribuição empíricas marginais $\tilde{F}_{n}^{1}$ e $\tilde{F}_{n}^{2}$ de $X$ e $Y, \operatorname{com} \tilde{F}_{n}^{1}(x)=\frac{1}{n} \sum_{k=1}^{n} \mathbb{1}\left\{x_{k} \leq x\right\}$ e $\tilde{F}_{n}^{2}(y)=\frac{1}{n} \sum_{k=1}^{n} \mathbb{1}\left\{y_{k} \leq y\right\}$. As pseudo-observações $\boldsymbol{U}_{1}, \boldsymbol{U}_{2}, \ldots, \boldsymbol{U}_{n}$ da cópula correspondente ao vetor aleatório $\boldsymbol{X}$ são definidas por

$$
\boldsymbol{U}_{i}=\left(U_{1 i}, U_{2 i}\right)=\left(\tilde{F}_{n}^{1}\left(X_{i}\right), \tilde{F}_{n}^{1}\left(Y_{i}\right)\right)
$$

para $i=1, \ldots, n$. Equivalentemente à Definição 3.3.1, a cópula empírica pode ser definida como a função de distribuição empírica $\tilde{F}_{n}$ das pseudo-observações $\boldsymbol{u}_{1}, \boldsymbol{u}_{2}, \ldots, \boldsymbol{u}_{n}$. 
Alternativamente, os polinômios de Bernstein,

$$
B_{i}^{m}(x)=\sum_{j=0}^{m} \tilde{F}_{n}^{i}\left(\frac{j}{m}\right)\left(\begin{array}{c}
m \\
j
\end{array}\right) x^{j}(1-x)^{m-j}
$$

serão utilizados para aproximar as distribuições marginais $F_{i}, i=1,2$, de $X$ e $Y$ e obter as pseudo-observações da cópula $C$. As pseudo-observações determinadas dessa forma serão usadas para obter uma cópula empírica $\hat{C}_{n}$ e a cópula de Bernstein correspondente será

$$
B_{\hat{C}_{n}}^{m}\left(u_{1}, u_{2}\right)=\sum_{j_{1}=0}^{m} \sum_{j_{2}=0}^{m} \hat{C}_{n}\left(\frac{j_{1}}{m}, \frac{j_{2}}{m}\right)\left(\begin{array}{c}
m \\
j_{1}
\end{array}\right) u_{1}^{j_{1}}\left(1-u_{1}\right)^{m-j_{1}}\left(\begin{array}{c}
m \\
j_{2}
\end{array}\right) u_{2}^{j_{2}}\left(1-u_{2}\right)^{m-j_{2}} .
$$

Desse modo, a aproximação sugerida nesse trabalho para a função de distribuição $F$ do vetor aleatório $\boldsymbol{X}$ é

$$
\hat{F}_{m, n}(x, y)=B_{\hat{C}_{n}}^{m}\left(B_{1}^{m}(x), B_{2}^{m}(y)\right)
$$

A função de probabilidade será obtida novamente utilizando a função $\Delta^{2}$.

\subsection{Avaliação das Estimativas}

Para avaliar a qualidade das estimativas sugeridas nas seções anteriores, os métodos descritos foram aplicados a conjuntos de dados simulados e comparados por meio do cálculo de distâncias entre as funções de probabilidade estimadas e a função de probabilidade teórica.

Considere o vetor aleatório $\boldsymbol{X}=(X, Y) \in \mathcal{X}_{1} \times \mathcal{X}_{2}$, com $\mathcal{X}_{i}=\left\{a_{i 1}, \ldots, a_{i r_{i}}\right\}, r_{i} \in \mathbb{N}$ e $i=1,2$. É interessante notar que a função de probabilidade pode ser escrita como uma matriz $\boldsymbol{Q}_{r_{1} \times r_{2}}$, onde o elemento $q_{(i, j)}=p\left(a_{1 i}, a_{2 j}\right)=P\left(X=a_{1 i}, Y=a_{2 j}\right), 1 \leq i \leq r_{1}$, $1 \leq j \leq r_{2}$. Além disso, note que o vetor $\boldsymbol{q}=\left(q_{(1,1)}, \ldots, q_{\left(1, r_{2}\right)}, q_{(2,1)}, \ldots, q_{\left(r_{1}, r_{j}\right)}\right)$ é um vetor composicional, isto é, um vetor que as somas das componentes é uma constante conhecida (Aitchison, 2003). No caso do vetor $\boldsymbol{q}$, as componentes $q_{(i, j)}$ são números no intervalo $[0,1]$ e $\sum_{i} \sum_{j} q_{(i, j)}=1$. A notação usualmente utilizada nesse caso é $\boldsymbol{q} \in \mathcal{S}^{R}$, onde $R=r_{1} . r_{2}$ e $\mathcal{S}^{R} \subset \mathbb{R}^{R}$ é o simplex $R$-dimensional. Considere a distância de Aitchison, definida a seguir.

Definição 3.4.1 Seja $\boldsymbol{q}_{1}$ e $\boldsymbol{q}_{2}$ dois vetores em $\mathbb{R}^{R}$, com $\boldsymbol{q}_{i}=\left(q_{i 1}, q_{i 2}, \ldots, q_{i R}\right)$ e $\sum_{j} q_{i j}=1$, $i=1,2$. A distância de Aitchison é definida por

$$
\delta\left(\boldsymbol{q}_{1}, \boldsymbol{q}_{2}\right)=\sqrt{\sum_{j=1}^{R}\left(L_{j}-\bar{L}\right)^{2}}
$$

onde $L_{j}=\ln \left(\frac{q_{1 j}}{q_{2 j}}\right), 1 \leq j \leq R$, e $\bar{L}=\frac{1}{R} \sum_{j=1}^{R} L_{j}$.

A distância de Aitchison tem uma expressão similar a um desvio-padrão (multiplicado pela constante $\sqrt{R-1}$ ). Equivalentemente, a distância de Aitchison pode ser escrita das seguintes maneiras: 


$$
\begin{gathered}
\delta\left(\boldsymbol{q}_{1}, \boldsymbol{q}_{2}\right)=\sqrt{\frac{1}{R} \sum_{i<j}\left(\log \frac{q_{1 i}}{q_{1 j}}-\log \frac{q_{2 i}}{q_{2 j}}\right)^{2}}=\sqrt{\frac{1}{R} \sum_{i<j}\left(\log \frac{q_{1 i}}{q_{2 i}}-\log \frac{q_{1 j}}{q_{2 j}}\right)^{2}}, \\
\delta\left(\boldsymbol{q}_{1}, \boldsymbol{q}_{2}\right)=\sqrt{\sum_{j=1}^{R}\left(\log \frac{q_{1 j}}{g\left(\boldsymbol{q}_{1}\right)}-\log \frac{q_{2 j}}{g\left(\boldsymbol{q}_{2}\right)}\right)^{2}},
\end{gathered}
$$

onde $g\left(\boldsymbol{q}_{i}\right)=\left\{q_{i 1} \cdot q_{i 2} \ldots q_{i R}\right\}^{\frac{1}{R}}$ é a média geométrica das componentes do vetor $\boldsymbol{q}_{i}, i=1,2$.

No trabalho de Aitchison (2008), ele explica a razão por utilizar $\log \left(q_{1 j} / q_{2 j}\right), j=1, \ldots, R$, da seguinte maneira: "vetores composicionais contém apenas informações sobre valores relativos de suas componentes, por isso primeiramente 'pensar em razões'. Então, ao perceber que os quocientes são mais difíceis de lidar do que somas ou diferenças, "pensar no log das razões'."

Antes de estudar as propriedades dessa distância, considere as seguintes operações:

- $c: \mathbb{R}^{R} \rightarrow \mathcal{S}^{R}$ definida por $c(\boldsymbol{x})=\frac{1}{\sum_{i} x_{i}} \boldsymbol{x}=\left(\frac{x_{1}}{\sum_{i} x_{i}}, \ldots, \frac{x_{R}}{\sum_{i} x_{i}}\right)$, com $\boldsymbol{x} \in \mathbb{R}^{R}$;

- $($ Operação perturbação $) \oplus: \mathcal{S}^{R} \times \mathcal{S}^{R} \rightarrow \mathcal{S}^{R}$ tal que $\boldsymbol{q} \oplus \boldsymbol{s}=c\left(q_{1} . s_{1}, \ldots q_{R} . s_{R}\right)$, com $\boldsymbol{q}, \boldsymbol{s} \in \mathcal{S}^{R}$

- $($ Operação potência $) \otimes: \mathbb{R} \times \mathcal{S}^{R} \rightarrow \mathcal{S}^{R}$ tal que $a \otimes \boldsymbol{q}=c\left(q_{1}^{a}, \ldots, q_{R}^{a}\right)$, com $a \in \mathbb{R}$ e $\boldsymbol{q} \in \mathcal{S}^{R}$.

As operações $\oplus \mathrm{e} \otimes$ podem ser vistas como análogas às operações de translação e multiplicação por escalar de vetores no espaço real. Assim, o espaço $\mathcal{S}^{R}$ pode ser visto como um espaço vetorial e a distância de Aitchison define uma métrica em $\mathcal{S}^{R}$ (Aitchison, 2008). Para $\boldsymbol{q}, \boldsymbol{r}, \boldsymbol{s} \in \mathcal{S}^{R}$, é possível mostrar que a distância de Aitchison $\delta$ satisfaz as exigências de uma métrica.

M1. Positividade: $\delta(\boldsymbol{q}, \boldsymbol{s})>0$, para $\boldsymbol{q} \neq \boldsymbol{s}$, e $\delta(\boldsymbol{q}, \boldsymbol{s})=0$, se $\boldsymbol{q}=\boldsymbol{s}$

M2. Simetria: $\delta(\boldsymbol{q}, \boldsymbol{s})=\delta(\boldsymbol{s}, \boldsymbol{q})$;

M3. Relação Potência: $\delta(a \otimes \boldsymbol{q}, a \otimes \boldsymbol{s})=|a| \delta(\boldsymbol{q}, \boldsymbol{s})$;

M4. Desigualdade Triangular: $\delta(\boldsymbol{q}, \boldsymbol{r})+\delta(\boldsymbol{r}, \boldsymbol{s}) \geq \delta(\boldsymbol{q}, \boldsymbol{s})$.

Note que a relação potência faz um papel análogo à multiplicação por escalar na distância euclidiana em $\mathbb{R}^{R}$. Além dessas, $\delta$ apresenta outras propriedades bastante interessantes, como

M5. Invariância sob permutações: $\delta(\boldsymbol{P} \boldsymbol{q}, \boldsymbol{P} \boldsymbol{s})=\delta(\boldsymbol{q}, \boldsymbol{s})$, para alguma matriz de permutação $P$

M6. Invariância sob perturbações: $\delta(\boldsymbol{q} \oplus \boldsymbol{r}, \boldsymbol{s} \oplus \boldsymbol{r})=\delta(\boldsymbol{q}, \boldsymbol{s})$;

\footnotetext{
${ }^{1}$ Tradução do autor.
} 
M7. Dominância sub-composicional: Seja $\boldsymbol{s}_{q}$ e $\boldsymbol{s}_{r}$ são sub-composições similares de $\boldsymbol{q}$ e $\boldsymbol{r}$, isto é, $\boldsymbol{s}_{q}=\left(q_{\pi_{1}}, q_{\pi_{2}}, \ldots, q_{\pi_{c}}\right)$ e $\boldsymbol{s}_{r}=\left(r_{\pi_{1}}, r_{\pi_{2}}, \ldots, r_{\pi_{c}}\right)$, com $C \leq R, \pi_{i} \in\{1, \ldots, R\}$, $\forall i$, e $\pi_{i} \neq \pi_{j}, i \neq j$. Então $\delta\left(\boldsymbol{s}_{q}, \boldsymbol{s}_{r}\right) \leq \delta(\boldsymbol{q}, \boldsymbol{r})$.

A propriedade de dominância sub-composicional é análoga à propriedade métrica em $\mathbb{R}^{R}$ que a distância euclidiana entre dois pontos não pode ser maior que a distância entre as projeções desses dois pontos (Aitchison, 2008).

Aitchison (2003, 2008) e Pawlowsky et al. (2007) fornecem outros argumentos afirmando que $\delta$ é a distância mais adequada no contexto de dados composicionais. Entretanto, para casos onde os vetores contém zeros, essa distância não está definida e é necessário fazer uma pequena adaptação. Assim, considere $\delta_{\epsilon}\left(\boldsymbol{q}_{1}, \boldsymbol{q}_{2}\right)=\delta\left(\frac{\boldsymbol{q}_{1}+\epsilon}{1+R \epsilon}, \frac{\boldsymbol{q}_{2}+\epsilon}{1+R \epsilon}\right)=\delta\left(c\left(\boldsymbol{q}_{1}+\epsilon\right), c\left(\boldsymbol{q}_{2}+\epsilon\right)\right)$, $\operatorname{com} \epsilon>0$ fixado. Uma aplicação dessa distância em um contexto um pouco diferente pode ser encontrada em Fossaluza et al. (2009). A razão de tal correção é que quando se utiliza a abordagem bayesiana em um contexto onde o parâmetro $\boldsymbol{\theta}=\left(\theta_{1}, \ldots \theta_{R}\right)$ é um vetor de proporções (isto é, um vetor composicional com $\sum \theta_{i}=1$ ), é comum adotar uma priori (não informativa) uniforme em $\mathcal{S}^{R}$, Dirichlet $(1, \ldots, 1)$. Nesse contexto, quando uma amostra $\left(n_{1}, \ldots n_{R}\right)$ de tamanho $N$ é observada, com $\sum n_{i}=N$, a posteriori obtida é Dirichlet $\left(n_{1}+1, \ldots, n_{R}+1\right)$. A estimativa pontual considerando a função de perda quadrática é $\hat{\boldsymbol{\theta}}=c\left(n_{1}+1, \ldots n_{R}+1\right)=\left(\frac{n_{1}+1}{N+R}, \ldots \frac{n_{R}+1}{N+R}\right)$. Deste modo, se a frequência de alguma casela for igual a zero, $n_{i}=0$, a estimativa de $\theta_{i}$ é $\frac{1}{N+R}$. Por uma razão análoga à do artigo citado, o valor de $\epsilon$ adotado aqui será $1 / n$, onde $n$ é número de observações completas utilizadas na estimação.

Para comparar as estimativas obtidas pelos métodos descritos nas seções anteriores, utilizar-se-á a distância de Aitchison. Além disso, algumas distâncias frequentemente utilizadas na literatura também serão apresentadas para comparação. São elas:

1. Distância euclidiana: $D_{1}(\boldsymbol{q}, \boldsymbol{s})=\left\{\sum_{i=1}^{R}\left(q_{i}-s_{i}\right)^{2}\right\}^{1 / 2}$;

2. Distância de variação total: $D_{2}(\boldsymbol{q}, \boldsymbol{s})=\frac{1}{2} \sum_{i=1}^{R}\left|q_{i}-s_{i}\right|$;

3. Divergência de Kullback-Leibler: $D_{3}(\boldsymbol{q}, \boldsymbol{s})=\frac{1}{2} \sum_{i=1}^{R} q_{i} \log \frac{q_{i}}{s_{i}}+\frac{1}{2} \sum_{i=1}^{R} s_{i} \log \frac{s_{i}}{q_{i}}$.

Note que a divergência de Kullback-Leibler (K-L) apresenta um problema similar à distância de Aitchison na presença de zeros. Deste modo, a mesma solução será utilizada e a divergência de K-L será calculada entre os vetores corrigidos $\boldsymbol{q}^{*}=c(\boldsymbol{q}+\epsilon)$ e $\boldsymbol{s}^{*}=c(\boldsymbol{s}+\epsilon)$. Para as distâncias euclidiana e de variação total é possível calcular as distâncias entre os vetores originais $\boldsymbol{q}$ e $\boldsymbol{s}$. Assim, serão apresentadas também as distâncias entre os vetores corrigidos $\boldsymbol{q}^{*}$ e $\boldsymbol{s}^{*}$ para avaliar o efeito dessa correção. 


\section{Capítulo 4}

\section{Aplicações}

Alice: "This is impossible!"

Chapeleiro: "Only if you believe it is..." (Alice in Worderland (2010))

Nas seções que seguem, são apresentados exemplos de aplicações das quatro soluções propostas no capítulo anterior para a estimação de funções de probabilidade bivariadas. As soluções foram resumidas por meio de gráficos e a comparação com a distribuição teórica foi baseada nas distâncias sugeridas na Seção 3.4. Foi utilizado o software R (R Development Core Team, 2010) e as rotinas computacionais podem ser encontradas no Apêndice A.

Antes da apresentação dos exemplos, é necessário introduzir alguns conceitos.

\subsection{Definições Preliminares}

Definição 4.1.1 A variável aleatória $Y$ tem distribuição beta-binomial com parâmetros $N$, a e b, onde $N \in \mathbb{N}, a, b \in \mathbb{R}_{+}$, se sua função de probabilidade é dada por

$$
p(y)=\left(\begin{array}{c}
N \\
y
\end{array}\right) \frac{B(a+y, b+N-y)}{B(a, b)}=\frac{\Gamma(N+1) \Gamma(a+y) \Gamma(b+N-y) \Gamma(a+b)}{\Gamma(y+1) \Gamma(N-y+1) \Gamma(a+b+N) \Gamma(a) \Gamma(b)},
$$

com $y \in\{0,1, \ldots, N\}, \Gamma$ é a função gama e $B$ é a função beta.

Definição 4.1.2 Considere $\boldsymbol{\rho}$ uma matriz de correlação. A cópula normal com parâmetro $\boldsymbol{\rho}$ pode ser escrita como

$$
C_{\boldsymbol{\rho}}(\boldsymbol{u})=\Phi_{\boldsymbol{\rho}}\left(\Phi^{-1}\left(u_{1}\right), \ldots, \Phi^{-1}\left(u_{k}\right)\right)
$$

onde $\boldsymbol{u}=\left(u_{1}, \ldots, u_{k}\right) \in I^{k}, \Phi^{-1}$ é a inversa da função de distribuição da normal univariada com média zero e variância 1 e $\Phi_{\boldsymbol{\rho}}$ é a função de distribuição normal multivariada com vetor de médias igual a $\mathbf{0}=(0, \ldots, 0)$ e matriz de covariância $\boldsymbol{\rho}$. 
Definição 4.1.3 A cópula de Gumbel bivariada é definida por

$$
C(\boldsymbol{u})=\exp \left\{-\left[\left(-\ln u_{1}\right)^{\theta}+\left(-\ln u_{2}\right)^{\theta}\right]^{\frac{1}{\theta}}\right\},
$$

com $\boldsymbol{u}=\left(u_{1}, u_{2}\right) \in I^{2}$ e $\theta \in[1, \infty)$. A cópula de Gumbel é uma cópula archimediana (vide exemplo 2.2.6) com função geradora $\varphi(t)=(-\ln t)^{\theta}$ (Nelsen, 2006).

\subsection{Exemplo 1: Distribuição Bivariada Simétrica}

Neste primeiro exemplo, foi construída uma distribuição discreta com marginais simétricas porém correlacionadas. Para isso, considerou-se que $X_{1}$ tem distribuição beta-binomial com parâmetros $N_{x}=15, a=5$ e $b=5$ (figura 4.1) e $Y_{1}$ tem distribuição binomial com parâmetros $N_{y}=10$ e $p=0,5$ (figura 4.2). A distribuição conjunta foi construída utilizando o Teorema de Sklar (Teorema 2.2.2), considerando a cópula normal com parâmetro $\rho=0.7$. A figura 4.3 representa as funções de distribuição e de probabilidade conjunta do vetor aleatório $\left(X_{1}, Y_{1}\right)$. Para verificar se existe ganho em estimar separadamente as distribuições marginais e a cópula como proposto na Seção 3.3.2, foi simulado um conjunto de dados com 1000 observações dessa distribuição, sendo que 600 observações foram pares completos, 200 observações tiveram os valores de $X_{1}$ omitidos e, nas demais, foram omitidos os valores de $Y_{1}$.

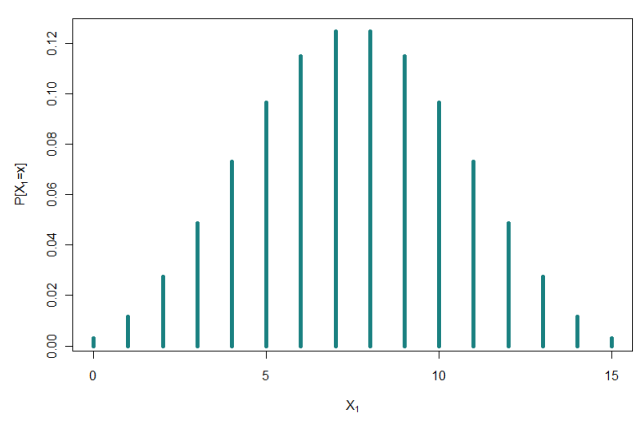

Figura 4.1: f.p. marginal de $X_{1}$

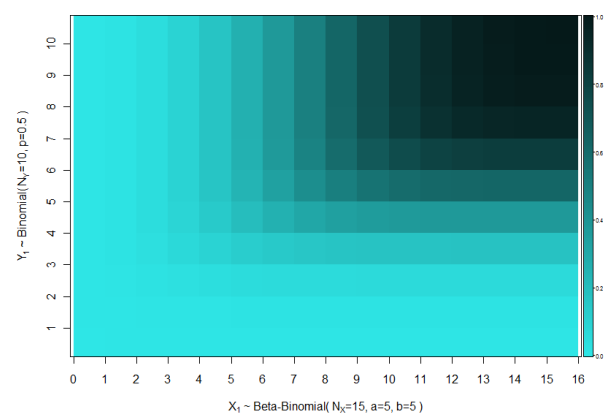

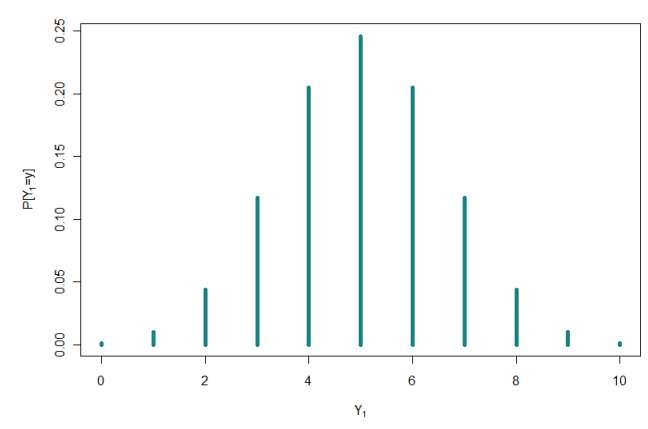

Figura 4.2: f.p. marginal de $Y_{1}$

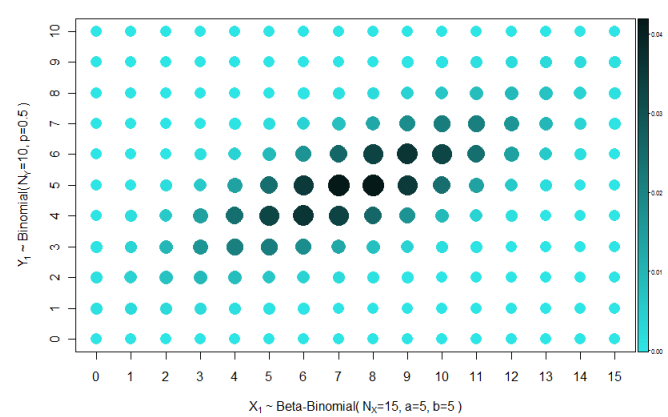

Figura 4.3: f.d. e f.p. teóricas de $\left(X_{1}, Y_{1}\right)$ 

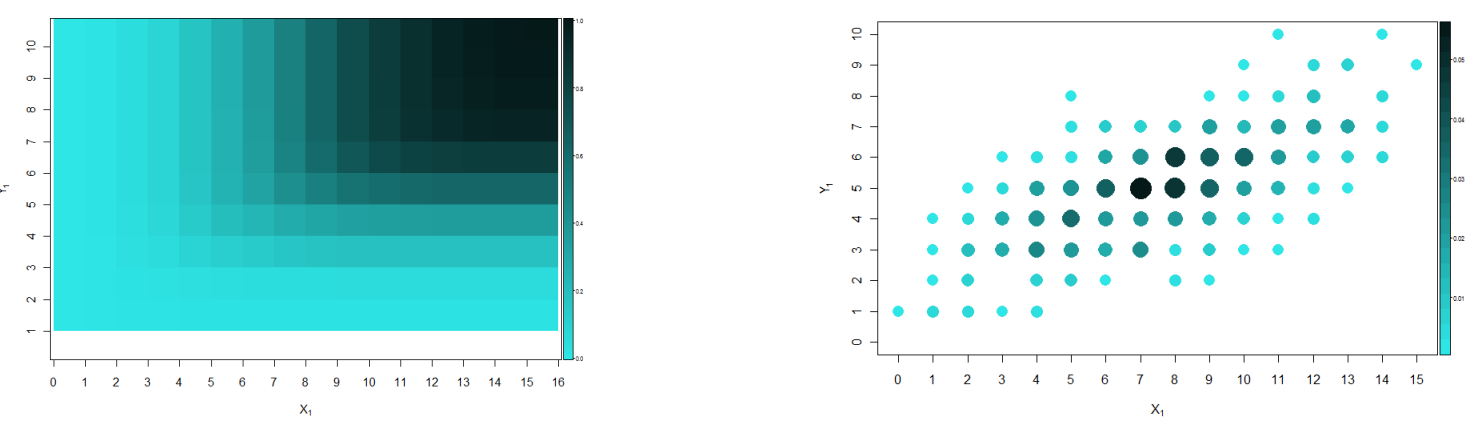

Figura 4.4: f.d. e f.p. empíricas
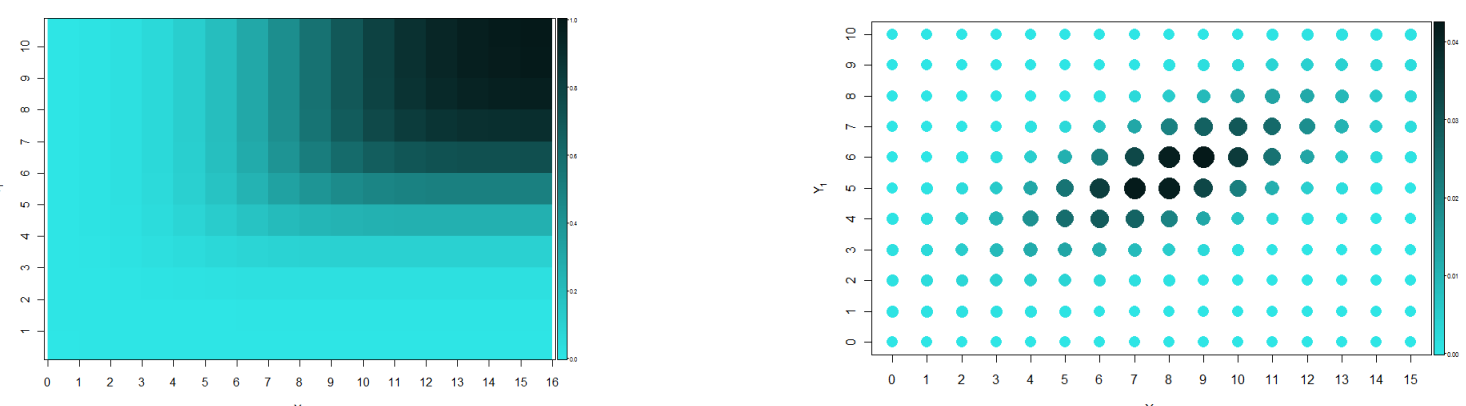

Figura 4.5: f.d. e f.p. estimadas pela Normal
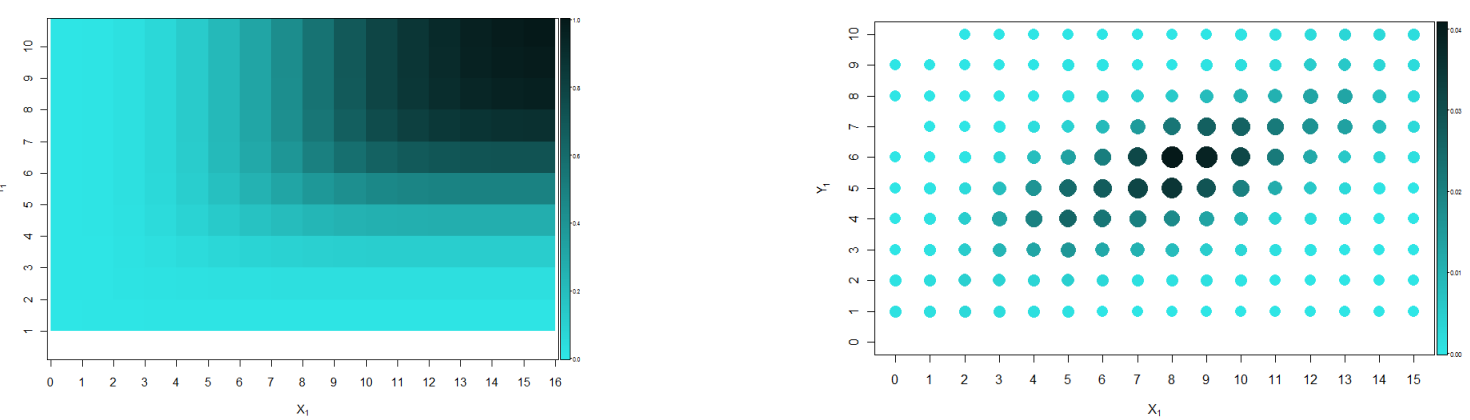

Figura 4.6: f.d. e f.p. estimadas pelo polinômio Bernstein
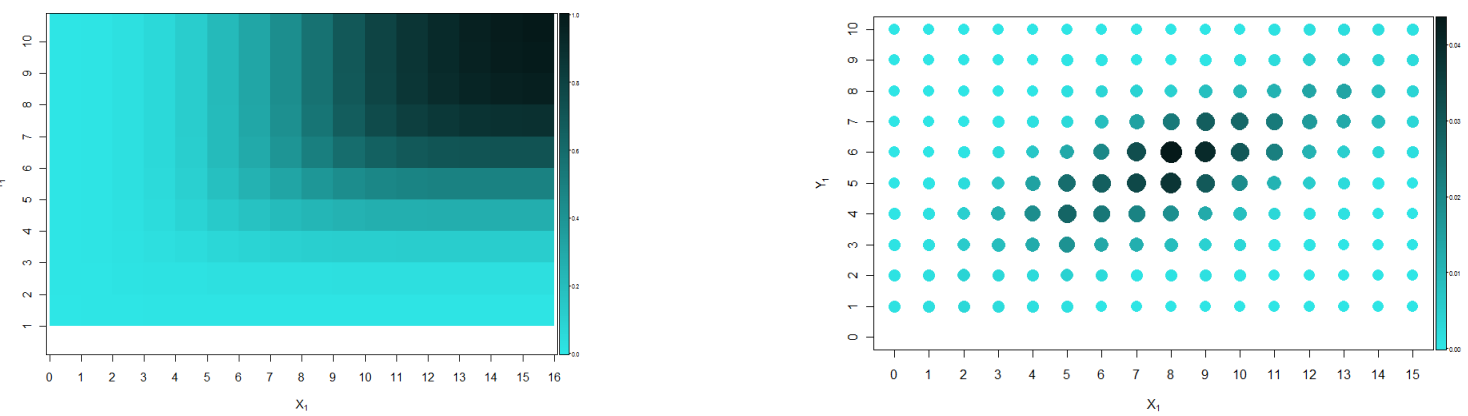

Figura 4.7: f.d. e f.p. estimadas pela cópula de Bernstein 
É possível notar, pelos gráficos 4.4 a 4.7, que a estimação pela cópula empírica é bastante limitada, devido à presença de zeros. A aproximação pela distribuição normal parece ter sido a melhor dentre as quatro propostas para esse caso. Isso acontece pois esse exemplo foi propositalmente construído utilizando distribuições marginais simétricas e a cópula normal, de modo que a distribuição conjunta seja simétrica e com uma forma semelhante à distribuição normal. A comparação entre essas estimativas é apresentada na tabela 4.1, onde os valores marcado com * representam as distâncias calculadas para cada vetor de estimativas modificado $\boldsymbol{q}^{*}=c(\boldsymbol{q}+\epsilon)$ e o vetor com a distribuição teórica modificado $\boldsymbol{s}^{*}=c(\boldsymbol{s}+\epsilon)$, $\operatorname{com} \epsilon=1 / 600$. Para as distâncias euclidiana e de variação total é possível notar que essa correção apenas muda a escala, preservando a ordenação das distâncias para cada vetor de estimativas original, $\boldsymbol{q}$, e o vetor com a distribuição teórica $\boldsymbol{s}$.

A estimação utilizando cópulas de Bernstein também parece ser razoável, tanto visualmente (figura 4.7) quanto considerando a distância de Aitchison (tabela 4.1). Contudo, a estimativa utilizando a distribuição empírica apresentou menores distâncias euclidiana e de variação total, mesmo que aparentemente essa estimativa seja a mais limitada. Isso é um indicativo de que a distância de Aitchison é mais adequada do que as distâncias usuais para a comparação de funções de probabilidade discretas.

\begin{tabular}{|l|c|c|c|c|c|c|}
\hline \multirow{2}{*}{ Método de Estimação } & \multicolumn{7}{|c|}{ Distância } \\
\cline { 2 - 7 } & Aitchison* & \multicolumn{2}{|c|}{ Euclidiana } & \multicolumn{2}{|c|}{ Variação Total } & K-L* $^{*}$ \\
\hline \hline Distribuição Empírica & 4.6298 & 0.0408 & $0.0316^{*}$ & 0.1362 & $0.1053^{*}$ & 0.0514 \\
Distribuição Normal & 3.1626 & 0.0355 & $0.0275^{*}$ & 0.1287 & $0.0995^{*}$ & 0.0335 \\
Polinômio de Bernstein & 3.8881 & 0.0414 & $0.0320^{*}$ & 0.1542 & $0.1192^{*}$ & 0.0461 \\
Cópula de Bernstein & 3.8286 & 0.0414 & $0.0320^{*}$ & 0.1535 & $0.1187^{*}$ & 0.0459 \\
\hline
\end{tabular}

Tabela 4.1: Comparação das estimativas do exemplo da Seção 4.2

\subsection{Exemplo 2: Distribuição Bivariada Assimétrica}

A distribuição que foi estimada neste exemplo foi construída como no exemplo anterior, contudo, além de apresentar dependência entre marginais, essa distribuição não é simétrica. Para isso, considerou-se que $X_{2}$ tem distribuição beta-binomial com parâmetros $N_{x}=15$, $a=0.85$ e $b=1.1$ (figura 4.8) e $Y_{2}$ tem distribuição binomial com parâmetros $N_{y}=10$ e $p=0,6$ (figura 4.9). A distribuição conjunta do vetor aleatório $\left(X_{2}, Y_{2}\right)$ (figura 4.10) foi construída considerando a cópula de Gumbel com parâmetro $\theta=2$. Novamente foi simulado um conjunto de dados com 1000 observações, sendo que 600 observações foram pares completos, 200 observações tiveram omitidos os valores de $X_{1}$ e, nas demais, foram omitidos os valores de $Y_{1}$. 


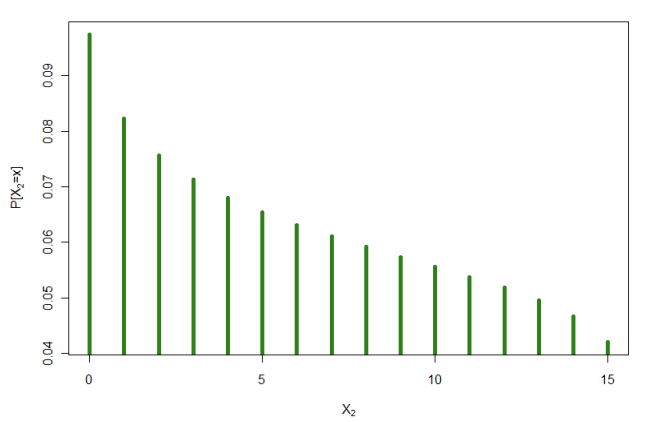

Figura 4.8: f.p. marginal de $X_{2}$

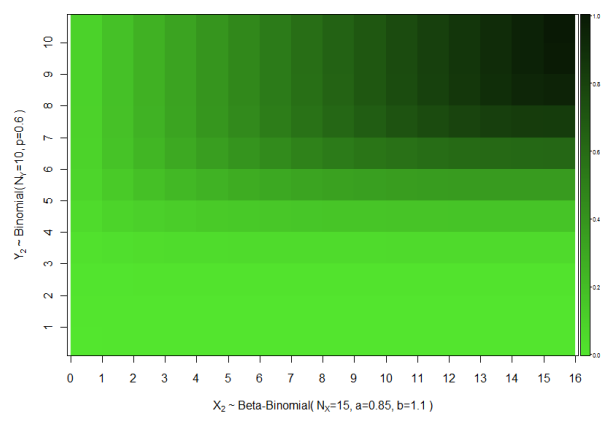

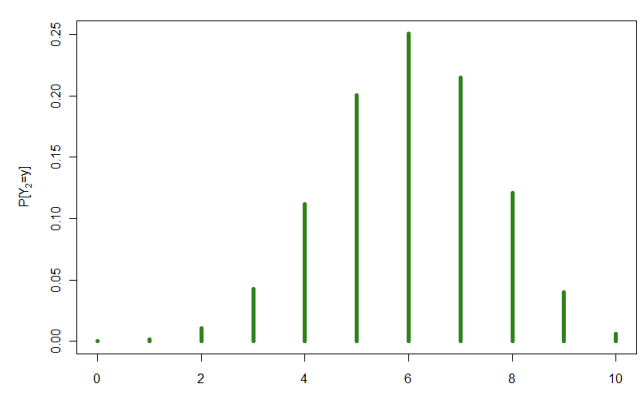

Figura 4.9: f.p. marginal de $Y_{2}$

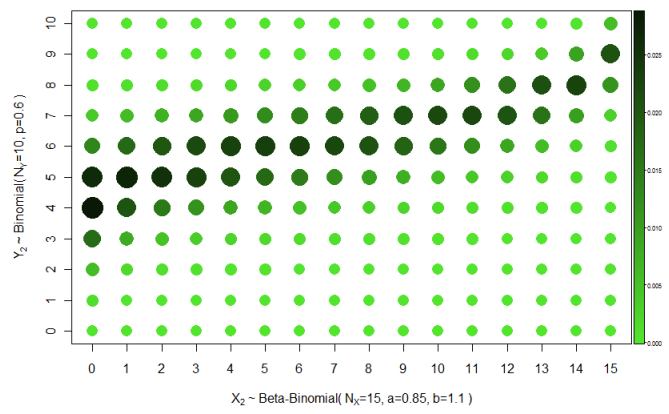

Figura 4.10: f.d. e f.p. teóricas de $\left(X_{2}, Y_{2}\right)$
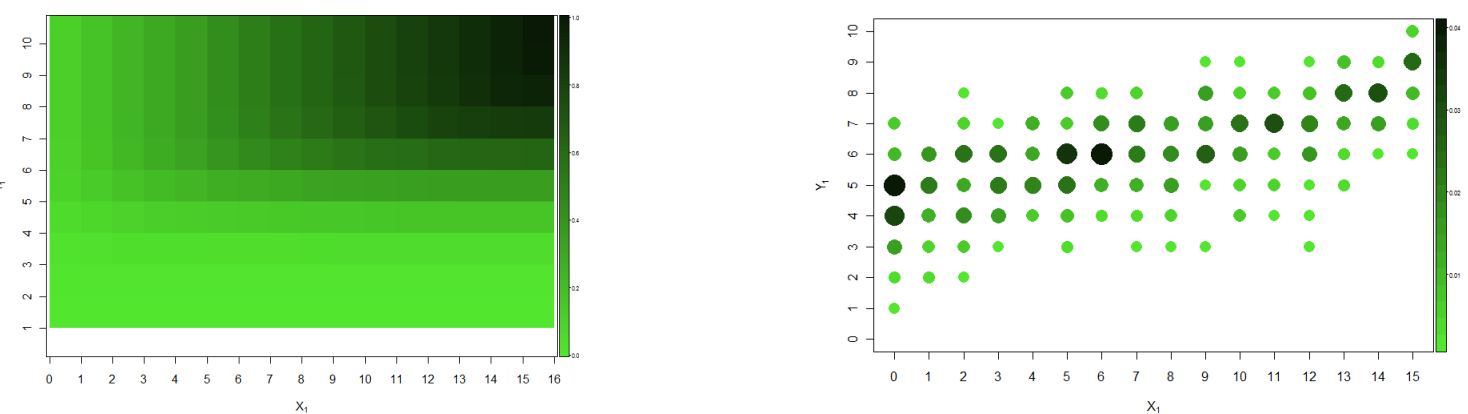

Figura 4.11: f.d. e f.p. empíricas
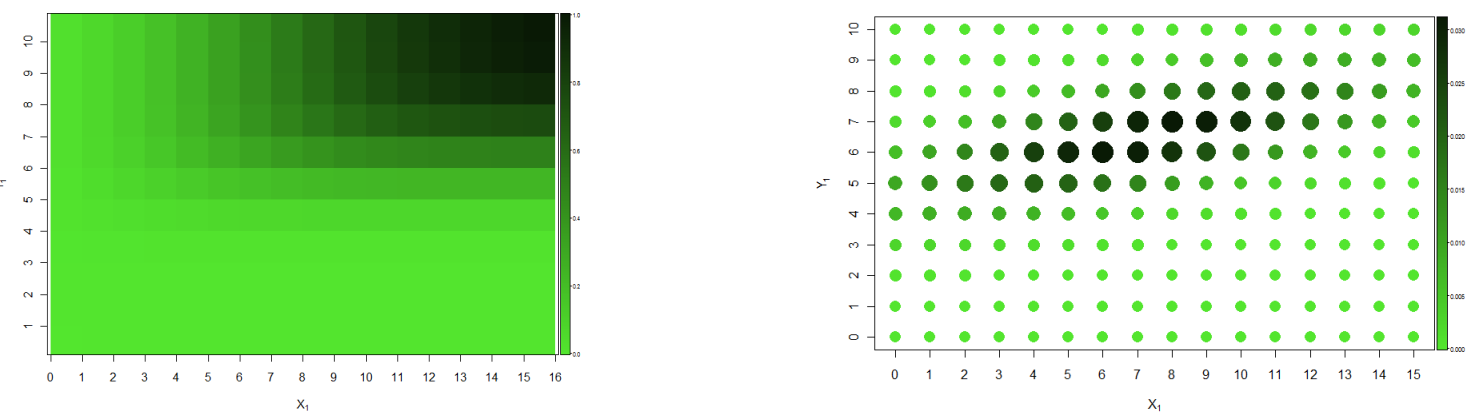

Figura 4.12: f.d. e f.p. estimadas pela Normal 

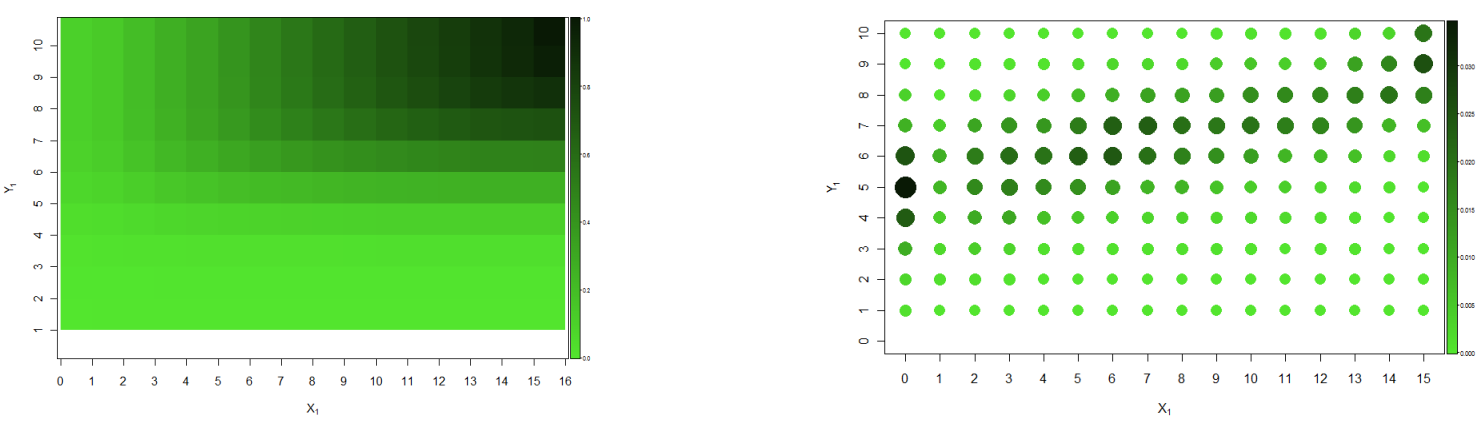

Figura 4.13: f.d. e f.p. estimadas pelo polinômio de Bernstein
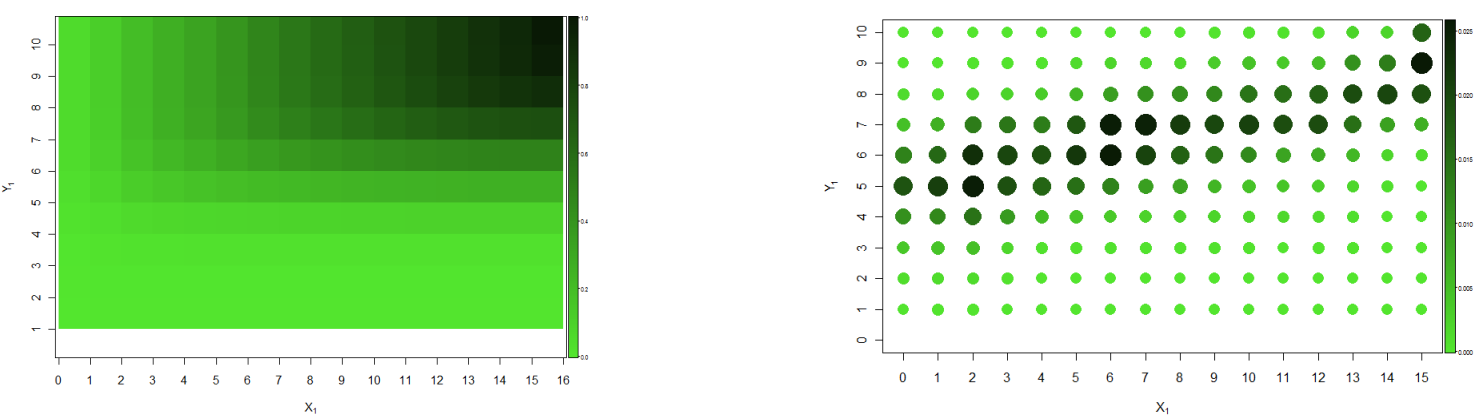

Figura 4.14: f.d. e f.p. estimadas pela cópula de Bernstein

Comparando o gráfico 4.11 com a distribuição teórica (figura 4.10), é possível notar que que diversos pontos foram estimados como zero. Além disso, as maiores estimativas ocorreram em torno dos pontos $(0,5)$ e $(6,6)$. Por apresentar uma forma simétrica, a estimação usando a distribuição normal concentrou as probabilidade em uma elipse em torno do ponto $(7,6)$ e parece não ser adequada nesse caso. É possível notar que a estimação utilizando o polinômio de Bernstein (figura 4.13 capturou a estrutura de dependência da distribuição, apesar de ter dado muita massa para o ponto $(0,5)$. Pelo gráfico 4.14, é possível notar que a estimação utilizando cópulas de Bernstein parece ter sido a melhor dentre as quatro propostas para esse caso. Isso acontece pois além de a cópula de Bernstein permitir captar diferentes estruturas de dependência, é possível utilizar toda a informação contida na amostra. A comparação entre essas estimativas é apresentada na tabela 4.2.

\begin{tabular}{|l|c|c|c|c|c|c|}
\hline \multirow{2}{*}{ Método de Estimação } & \multicolumn{7}{|c|}{ Distância } \\
\cline { 2 - 7 } & Aitchison* & \multicolumn{2}{|c|}{ Euclidiana } & \multicolumn{2}{|c|}{ Variação Total } & K-L $^{*}$ \\
\hline \hline Distribuição Empírica & 4.5442 & 0.0456 & $0.0352^{*}$ & 0.1679 & $0.1298^{*}$ & 0.0598 \\
Distribuição Normal & 5.7311 & 0.0624 & $0.0482^{*}$ & 0.2268 & $0.1754^{*}$ & 0.1137 \\
Polinômio de Bernstein & 4.6377 & 0.0481 & $0.0372^{*}$ & 0.1790 & $0.1384^{*}$ & 0.0725 \\
Cópula de Bernstein & 4.1397 & 0.0419 & $0.0324^{*}$ & 0.1512 & $0.1169^{*}$ & 0.0553 \\
\hline
\end{tabular}

Tabela 4.2: Comparação das estimativas do exemplo da Seção 4.3 


\subsection{Exemplo 3: Dados de Pacientes Obsessivos-Compulsivos}

Este exemplo foi apresentado na introdução e motivou o desenvolvimento desse trabalho. A seguir, são apresentados gráficos que resumem o conjunto de dados provenientes de um estudo com pacientes com transtorno obsessivo-compulsivo, realizado no IPq-HC-FMUSP. Vamos chamar de $X$ a variável $M$-YBOCS inicial e de $Y$ a variável $M$-YBOCS final. Nesse conjunto de dados há 1001 observações para $X$ e apenas 213 observações para $Y$.

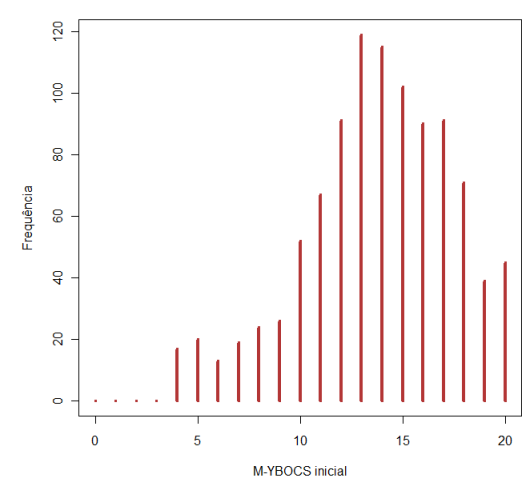

Figura 4.15: Frequências observadas de $X$

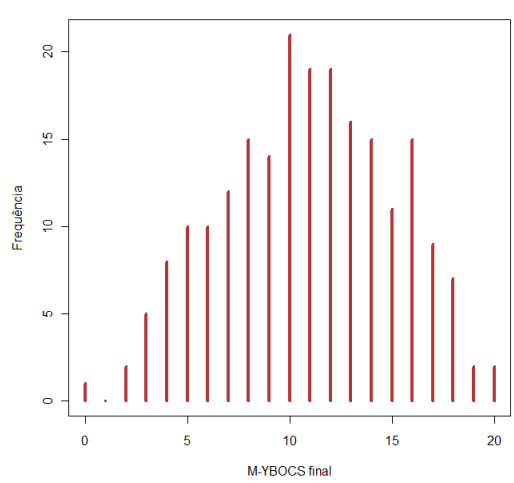

Figura 4.16: Frequências observadas de $Y$

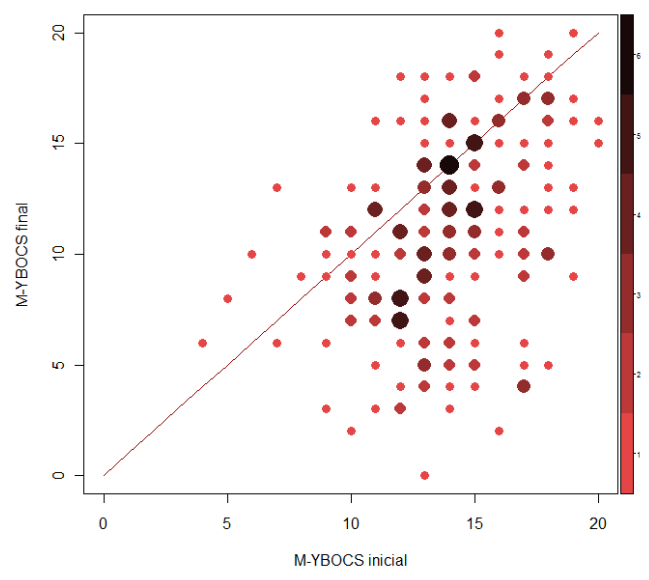

Figura 4.17: Frequências observadas para o par $(X, Y)$

Novamente, a estimação pela distribuição normal parece não captar a assimetria do conjunto de dados (figura 4.19). Pelos gráficos 4.20 e 4.21, é possível notar que as duas estimativas que utilizam polinômios de Bernstein parecem ter captado melhor a estrutura de dependência assimétrica apresentada pelo conjunto de dados. Como não é possível fazer uma comparação com uma distribuição teórica, aqui desconhecida, serão apresentadas as comparações entre as estimativas e a função de probabilidade empírica (tabela 4.3). A estimação utilizando a cópula de Bernstein foi a que mais se aproximou dos dados observados, segundo todas as distâncias utilizadas. 

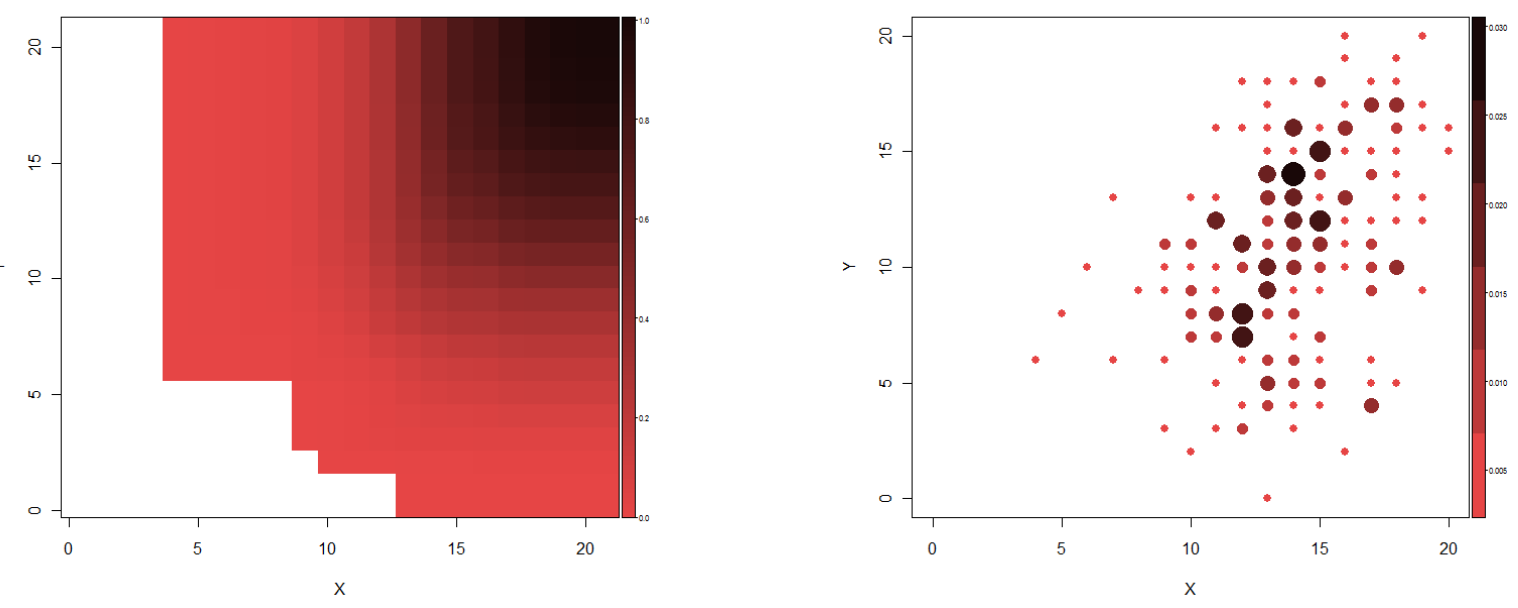

Figura 4.18: f.d e f.p. empíricas
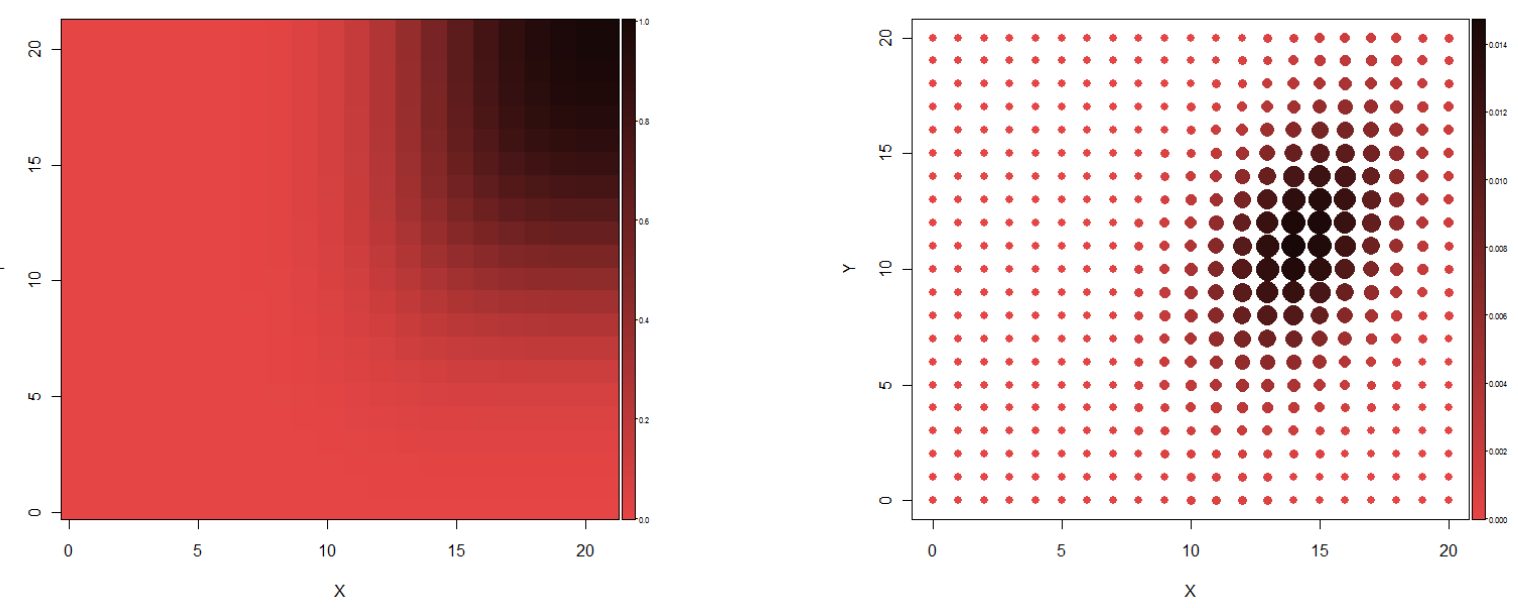

Figura 4.19: f.d. e f.p. estimadas pela Normal
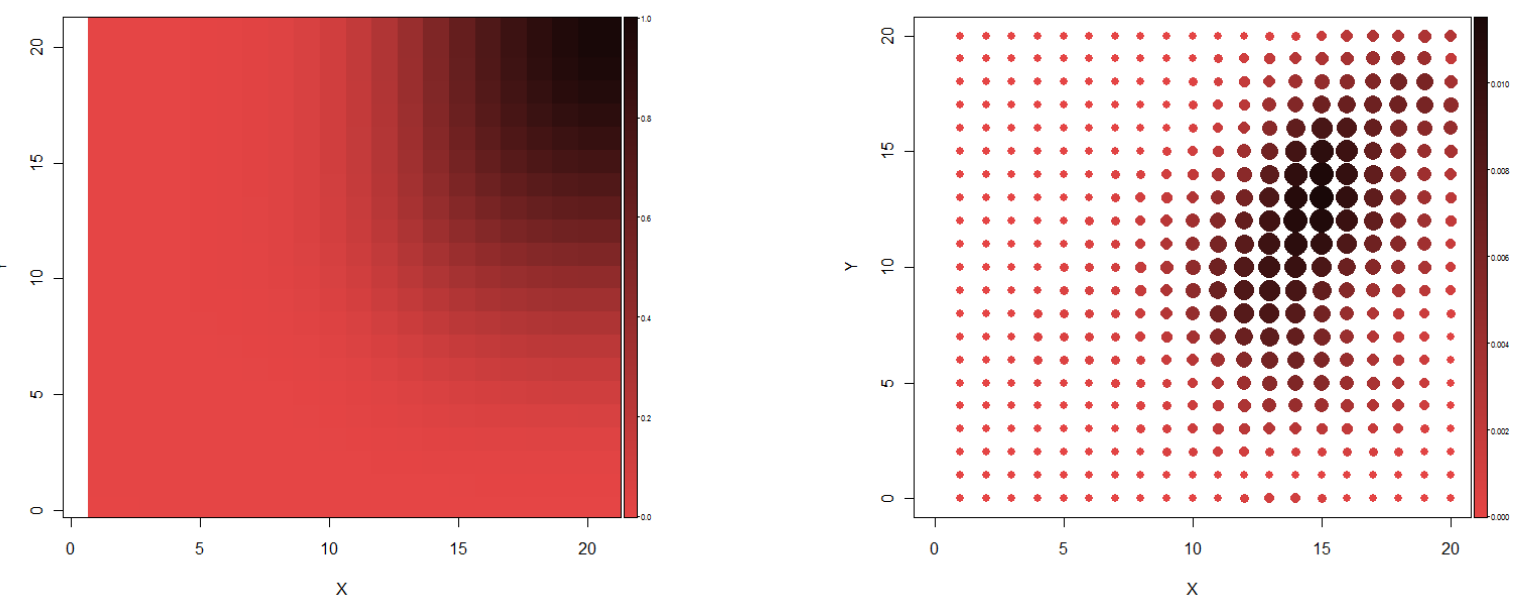

Figura 4.20: f.d. e f.p. estimadas pelo polinômio Bernstein 

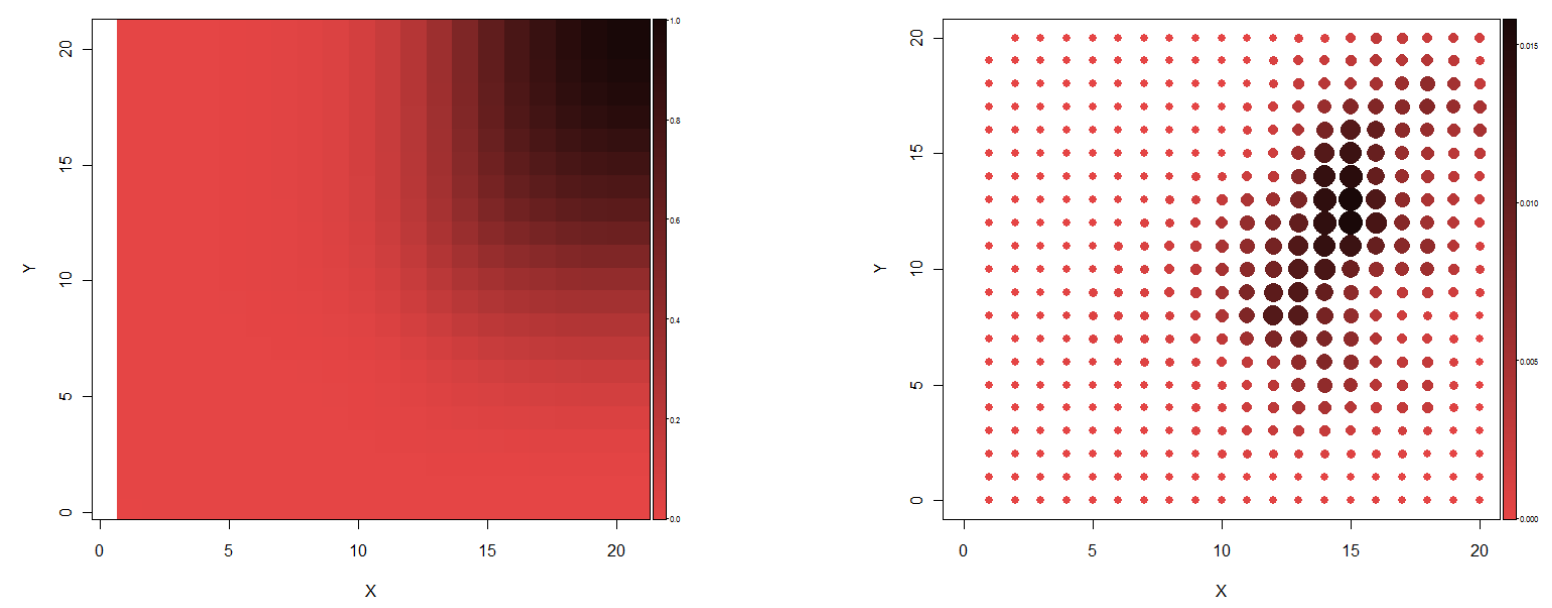

Figura 4.21: f.d e f.p. estimadas pela cópula de Bernstein

\begin{tabular}{|l|c|c|c|c|c|c|}
\hline \multirow{2}{*}{ Método de Estimação } & \multicolumn{6}{|c|}{ Distância } \\
\cline { 2 - 7 } & Aitchison* & \multicolumn{2}{|c|}{ Euclidiana } & \multicolumn{2}{|c|}{ Variação Total } & K-L* $^{*}$ \\
\hline \hline Distribuição Normal & 6.8134 & 0.0702 & $0.0229^{*}$ & 0.3999 & $0.1302^{*}$ & 0.0726 \\
Polinômio de Bernstein & 6.5709 & 0.0688 & $0.0224^{*}$ & 0.4012 & $0.1307^{*}$ & 0.0681 \\
Cópula de Bernstein & 6.2672 & 0.0644 & $0.0210^{*}$ & 0.3686 & $0.1201^{*}$ & 0.0610 \\
\hline
\end{tabular}

Tabela 4.3: Comparação das estimativas do exemplo da Seção 4.4

\subsection{Nota sobre o grau do polinômio de Bernstein}

Não existe na literatura uma fórmula geral para determinar qual grau ótimo $m^{*}$ do polinômio de Bernstein deve-se utilizar. Para o caso univariado, Babu et al. (2002) sugere, baseado em resultados empíricos, a utilização do grau $m=n / \log (n)$ e Petrone (1999a) sugere utilizar uma distribuição a priori para $m$ (em seu trabalho ela utiliza distribuições de Poisson e uniforme, ambas truncadas no valor 1000).

Nos exemplos das seções 4.2 e 4.3, temos uma amostra de tamanho $n=1000$. Todavia, temos dados perdidos para 200 observações na primeira variável e outras 200 observações para a outra variável e todas essas são utilizadas no método de estimação sugeridos. Nesse caso, não existe nenhuma sugestão na literatura sobre como determinar o grau ótimo do polinômio de Bernstein. Além disso, a sugestão de Babu et al. (2002) fica um pouco confusa, uma vez que existem dados faltantes. Se for considerada uma amostra de tamanho $n=$ 1000 , teríamos $m=120$. Se o tamanho amostral considerado for somente das observações completas $(n=600)$ usadas para estimar a cópula, teríamos $m=94$. Nesses exemplos em que temos a distribuição teórica, é possível fazer uma comparação das estimativas obtidas com a distribuição que está sendo estimada para diferentes valores de $m$. As tabelas 4.4 e 4.5, apresentam distâncias entre a distribuição teórica e as estimativas para diferentes valores de $m$. 
Na tabela 4.5, é possível notar que a partir de um certo valor de $m$, as estimativas começam a se distanciar da distribuição teórica. Isso ocorre pois como a cópula "verdadeira" é desconhecida e a cópula empírica é usada no polinômio de Bernstein, este converge para a cópula empírica. Assim, para que as estimativas se aproximem mais da distribuição teórica, além de aumentar o valor de $m$, é necessário aumentar o valor do tamanho amostral $n$.

Para os exemplos apresentados nas seções 4.2 e 4.3, utilizou-se $m=100$, que é aproximadamente $n / \log (n) \approx 94$, com $n=600$ sendo o número de observações completas. Pela mesma razão, no exemplo com dados reais considerou-se $m=40$.

\begin{tabular}{|r|c|c|c|c|c|c|}
\hline \multirow{2}{*}{$m$} & \multicolumn{6}{|c|}{ Distância } \\
\cline { 2 - 7 } & Aitchison* & \multicolumn{2}{|c|}{ Euclidiana } & \multicolumn{2}{|c|}{ Variação Total } & K-L $^{*}$ \\
\hline \hline 10 & 5.1625 & 0.0511 & 0.0395 & 0.2050 & 0.1585 & 0.0779 \\
20 & 3.7602 & 0.0451 & 0.0348 & 0.1648 & 0.1274 & 0.0480 \\
50 & 3.8685 & 0.0408 & 0.0316 & 0.1507 & 0.1165 & 0.0447 \\
80 & 3.8739 & 0.0433 & 0.0335 & 0.1559 & 0.1206 & 0.0464 \\
100 & 3.8286 & 0.0414 & 0.0320 & 0.1535 & 0.1187 & 0.0459 \\
200 & 3.8068 & 0.0408 & 0.0316 & 0.1511 & 0.1168 & 0.0446 \\
500 & 3.7644 & 0.0407 & 0.0314 & 0.1500 & 0.1160 & 0.0433 \\
1000 & 3.7876 & 0.0410 & 0.0317 & 0.1513 & 0.1170 & 0.0439 \\
1500 & 3.7808 & 0.0409 & 0.0316 & 0.1505 & 0.1163 & 0.0435 \\
2000 & 3.8026 & 0.0406 & 0.0314 & 0.1504 & 0.1163 & 0.0437 \\
5000 & 3.8180 & 0.0403 & 0.0315 & 0.1507 & 0.1165 & 0.0439 \\
\hline
\end{tabular}

Tabela 4.4: Comparação das estimativas para diferentes valores de $m$ no exemplo da Seção 4.2

\begin{tabular}{|r|c|c|c|c|c|c|}
\hline \multirow{2}{*}{$m$} & \multicolumn{7}{|c|}{ Distância } \\
\cline { 2 - 7 } & Aitchison* & \multicolumn{2}{|c|}{ Euclidiana } & \multicolumn{2}{|c|}{ Variação Total } & K-L $^{*}$ \\
\hline \hline 10 & 5.4566 & 0.0501 & 0.0387 & 0.2158 & 0.1668 & 0.0905 \\
20 & 4.9708 & 0.0460 & 0.0356 & 0.1843 & 0.1425 & 0.0738 \\
50 & 4.3093 & 0.0458 & 0.0354 & 0.1765 & 0.1365 & 0.0629 \\
80 & 4.4106 & 0.0441 & 0.0341 & 0.1663 & 0.1286 & 0.0621 \\
100 & 4.1397 & 0.0419 & 0.0324 & 0.1512 & 0.1169 & 0.0553 \\
200 & 4.3299 & 0.0438 & 0.0339 & 0.1625 & 0.1257 & 0.0602 \\
500 & 4.2635 & 0.0432 & 0.0334 & 0.1594 & 0.1232 & 0.0587 \\
1000 & 4.2616 & 0.0438 & 0.0339 & 0.1614 & 0.1248 & 0.0594 \\
1500 & 4.2297 & 0.0428 & 0.0331 & 0.1577 & 0.1219 & 0.0574 \\
2000 & 4.2390 & 0.0431 & 0.0333 & 0.1572 & 0.1215 & 0.0579 \\
5000 & 4.2581 & 0.0432 & 0.0334 & 0.1588 & 0.1228 & 0.0584 \\
\hline
\end{tabular}

Tabela 4.5: Comparação das estimativas para diferentes valores de $m$ no exemplo da Seção 4.3 


\section{Capítulo 5}

\section{Conclusões}

Creedy: "Die! Die! Why won't you die? Why won't you die?"

$\mathrm{V}$ : "Beneath this mask there is more than flesh. Beneath this mask there is an idea, Mr. Creedy, and ideas are bulletproof."

(V for Vendetta, Alan Moore e David Lloyd)

Nesse trabalho foi apresentada uma proposta para estimar a função de probabilidade de variáveis aleatórias discretas na presença de dados parcialmente observados e frequências iguais a zero. O método proposto utiliza cópulas de Bernstein e baseia-se na função de distribuição empírica. Além do método proposto, outros três métodos foram apresentados e a comparação dessas soluções foi feita utilizando uma distância adequada.

Outra forma bastante natural de abordar o problema de estimação de distribuições discretas considerando somente os dados completos é supor que, condicional ao conhecimento de $\boldsymbol{\theta}=\left(\theta_{11}, \theta_{12}, \ldots, \theta_{r_{1} r_{2}}\right), \boldsymbol{X}=(X, Y)$ tem distribuição multinomial, isto é, $\boldsymbol{X} \mid \boldsymbol{\theta} \sim \operatorname{Multinomial}(n, \boldsymbol{\theta})$, onde $\theta_{i, j}=P\left(X=a_{i}, Y=b_{j}\right),\left(a_{i}, b_{j}\right) \in \mathcal{X}_{1} \times \mathcal{X}_{2}, i \in\left\{1, \ldots, r_{1}\right\}$, $j \in\left\{1, \ldots, r_{2}\right\}$ e $n \in \mathbb{N}$ é um número fixado. Considere que a amostra observada foi $\left(x_{11}, x_{12}, \ldots, x_{r_{1} r_{2}}\right)$, onde $x_{i j}$ representa a frequência de observações da $i$-ésima categoria de $X$ e da $j$-ésima categoria de $Y$. O estimador de máxima verossimilhança para cada $\theta_{i j}$ seria $\hat{\theta}_{i j}=x_{i j} / n$, onde $n=\sum_{i} \sum_{j} x_{i j}$ é o tamanho da amostra completa. Esse estimador apresenta o mesmo problema que a função de distribuição empírica: se o par $\left(a_{i}, b_{j}\right)$ não foi observado na amostra, o parâmetro $\theta_{i j}$ correspondente seria estimado como zero. Um problema similar ocorre se a estimação for realizada sob a abordagem bayesiana. Se $n$ é o tamanho da amostra, $k-1=r_{1} \cdot r_{2}-1$ é a dimensão do espaço paramétrico e a priori não informativa usualmente utilizada Dirichlet $(1,1, \ldots, 1)$ for adotada, os parâmetros das categorias não observadas na amostra serão igualmente estimadas como 0 quando a perda 0-1 é considerada (a estimativa de Bayes é a moda da posteriori e, nesse exemplo, coincide com o estimador de máxima verossimilhança). Se a perda quadrática for escolhida, a estimativa de Bayes é a média da posteriori e, nesse caso, os parâmetros correspondentes às categorias não observadas serão igualmente estimadas como $1 /(n+k)$. Na presença de alguma informação 
prévia que levaria à adoção de uma distribuição a priori diferente, esse problema possivelmente não ocorreria.

Os dados observados incompletos também trazem alguma informação sobre o parâmetro e não devem ser desprezados. Supondo ainda o modelo multinomial, também é possível incluir essa informação parcial. Considere um exemplo bastante simples onde $X$ e $Y$ são variáveis de Bernoulli e deseja-se estimar sua distribuição conjunta, levando-se em conta também os dados que foram observados apenas para uma dessas variáveis. Denote por $\left(x_{0 *}, x_{1 *}\right)$ as observações realizadas somente para a variável $X$ e $\left(x_{* 0}, x_{* 1}\right)$ as observações feitas somente para $Y$. A verossimilhança para esse caso é

$$
V(\boldsymbol{\theta}) \propto \theta_{00}^{x_{00}} \theta_{01}^{x_{01}} \theta_{10}^{x_{10}} \theta_{11}^{x_{11}}\left(\theta_{00}+\theta_{01}\right)^{x_{0 *}}\left(\theta_{10}+\theta_{11}\right)^{x_{1 *}}\left(\theta_{00}+\theta_{10}\right)^{x_{* 0}}\left(\theta_{01}+\theta_{11}\right)^{x_{* 1}}
$$

Para exemplificar a importância de considerar também os dados parcialmente observados, suponha que a única observação completa foi $x_{00}=1$ e, além dessa, foram obtidas duas observações parciais $x_{0 *}=1$ e $x_{* 1}=1$. Se considerarmos somente a observação completa, a estimativa de máxima verossimilhança para o parâmetro $\boldsymbol{\theta}=\left(\theta_{00}, \theta_{01}, \theta_{10}, \theta_{11}\right)$ seria $\hat{\boldsymbol{\theta}}=(1,0,0,0)$. Claramente, pelo menos um dos parâmetros $\theta_{01}$ e $\theta_{11}$ não deveria ser estimado como zero nesse caso, uma vez que houve uma observação proveniente de uma das duas categorias correspondentes. A função de verossimilhanças considerando a informação marginal é $V(\boldsymbol{\theta}) \propto \theta_{00}\left(\theta_{00}+\theta_{01}\right)\left(\theta_{01}+\theta_{11}\right)$ e a estimativa de verossimilhança para esse caso é $\hat{\boldsymbol{\theta}}=(0.5,0.5,0,0)$, justificando a necessidade da inclusão dos dados parcialmente observados.

Suponha agora o caso mais geral onde as distribuições marginais de $X$ e $Y$ são multinomiais com parâmetros desconhecidos $\boldsymbol{\theta}_{1}=\left(\theta_{1 *}, \ldots, \theta_{r_{1 *}}\right)$ e $\boldsymbol{\theta}_{2}=\left(\theta_{* 1}, \ldots, \theta_{* r_{2}}\right)$, respectivamente, e o objetivo é estimar a distribuição conjunta, isto é, estimar o parâmetro $\boldsymbol{\theta}=\left(\theta_{11}, \theta_{12}, \ldots, \theta_{r_{1} r_{2}}\right)$. Suponha que a amostra $\left(x_{11}, x_{12}, \ldots, x_{r_{1} r_{2}}\right),\left(x_{1 *}, x_{2 *}, \ldots, x_{r_{1} *}\right)$, $\left(x_{* 1}, x_{* 2}, \ldots, x_{* r_{2}}\right)$ foi observada, onde $x_{i j}$ são as observação completas, $x_{i *}$ são as observação realizadas somente para a variável $X$ e $x_{* j}$ são observações realizadas somente para $Y$, com $i \in\left\{1, \ldots, r_{1}\right\}$ e $j \in\left\{1, \ldots, r_{2}\right\}$. A função de verossimilhanças para esse exemplo seria

$$
V(\boldsymbol{\theta}) \propto\left(\prod_{i=1}^{r_{1}} \prod_{j=1}^{r_{2}} \theta_{i j}^{x_{i, j}}\right)\left(\prod_{i=1}^{r_{1}} \theta_{i *}^{x_{i *}}\right)\left(\prod_{j=1}^{r_{2}} \theta_{* j}^{x_{* j}}\right),
$$

$\operatorname{com} \theta_{i *}=\sum_{j} \theta_{i, j}, i \in\left\{1, \ldots, r_{1}\right\}, \theta_{* j}=\sum_{i} \theta_{i, j}, j \in\left\{1, \ldots, r_{2}\right\}$ e $\sum_{i} \sum_{j} \theta_{i, j}=1$. Em geral, considerar os dados parcialmente observados dessa forma torna o problema de encontrar o EMV analiticamente intratável quando o tamanho da amostra ou o número de caselas aumenta (como é o caso dos exemplos apresentados no capítulo 4). Dependendo do tamanho da amostra e do número de caselas, a solução computacional também pode ser bastante complicada (e muitas vezes inviável). Além disso, essa abordagem pode conduzir à uma verossimilhança que não tem um único ponto de máximo de modo que o EMV não é único. 
Uma alternativa para considerar dados parcialmente observados é utilizar métodos computacionais como o algoritmo EM (Jensen e Nielsen, 2007). Uma solução bayesiana bastante elegante para esse problema, considerando uma reparametrização adequada, foi apresentada em Basu e Pereira (1982) e generalizada para tabelas maiores em Paulino e Pereira (1992, 1995). Sob a abordagem proposta nesses trabalhos, as distribuições condicionais dos novos parâmetros são Dirichlet independentes e, assim, a estimação pontual pode ser obtida. Contudo, independente da utilização da abordagem frequentista ou bayesiana, essa solução também fica limitada pela estrutura paramétrica da distribuição multinomial.

O método proposto para estimar distribuições discretas não apresenta as dificuldades descritas acima. A utilização das cópulas de Bernstein "corrige" o problema dos eventos raros, fornecendo estimativas da função de probabilidade também para os pontos não observados na amostra. Essa dificuldade é muito frequente na prática estatística pois na maior parte dos problemas aplicados a amostra disponível é relativamente pequena. A presença de dados perdidos, também muito frequente em aplicações e que pode gerar grande dificuldade nos métodos usuais de estimação, não é uma limitação ao método proposto e a informação trazida por observações parciais é facilmente incorporada na estimação. Além disso, quando uma distribuição paramétrica é utilizada, a estrutura de dependência entre as variáveis fica restrita à estrutura funcional daquela distribuição. Esse fato não ocorre no método proposto pois se trata de um alisamento da distribuição empírica e suporta qualquer estrutura de dependência sugerida pelos dados observados.

A boa performance do método proposto é verificada visualmente nos gráficos apresentados no capítulo 4 e comprovada pela distância de Aitchison. Exceto no exemplo 1, onde a distribuição teórica é simétrica e a aproximação pela distribuição normal apresenta menor distância em relação à distribuição teórica, o método sugerido forneceu melhores estimativas do que os demais métodos apresentados, independente da distância considerada. Contudo, pelo primeiro exemplo (seção 4.2), a distância de Aitchison parece ser melhor que as distâncias euclidiana e de variação total para a comparação de funções de probabilidade discretas.

O método também apresenta algumas limitações. Por exemplo, existe uma suposição que é feita implicitamente quando é utilizado um alisamento da função de distribuição empírica no caso de variáveis categóricas: quando há um ponto $\boldsymbol{x}_{0}$ no interior do domínio do vetor aleatório $\boldsymbol{X}$ que não foi observado na amostra, a função de distribuição desse ponto é aproximada por alguma quantidade entre os valores da função de distribuição calculada nos pontos vizinhos de $\boldsymbol{x}_{0}$. Isso implica que a função de probabilidade desse ponto provavelmente será diferente de zero e isso pode não ser verdade se a função de probabilidade a ser estimada não apresentar uma certa "suavidade". Entretanto, em alguns casos, não existem motivos aparentes que nos levem a acreditar que algum dos possíveis pontos do espaço amostral tenha probabilidade zero, mesmo que esse ponto não tenha sido observado na amostra, como no exemplo apresentado com dados do M-YBOCS. 


\subsection{Sugestões para Pesquisas Futuras}

Apesar de técnicas bastante similares ao método proposto já terem sido utilizadas para estimar distribuições multivariadas no caso contínuo (Babu e Chaubey, 2006; Durrleman et al., 2000b), nenhum outro trabalho no qual cópulas de Bernstein são utilizadas para aproximar distribuições discretas foi encontrado pelo autor. Desse modo, estudos nessa área ainda são necessários para avaliar as propriedades teóricas do método desenvolvido nessa tese.

Apesar da generalização do método para dimensões maiores $(k>2)$ parecer natural, ainda é necessário um estudo mais detalhado. Além disso, a utilização de outras funções de alisamento poderia ser considerada.

Do ponto de vista frequentista, uma possibilidade é estudar as propriedades assintóticas do método quando o tamanho amostral $n$ e o grau do polinômio de Bernstein $m$ aumentam.

Sob a perspectiva bayesiana, uma possibilidade é avaliar a existência de uma função de perda que torna o método um procedimento ótimo sob o enfoque da teoria da decisão. Ainda é possível tentar encontrar um par ótimo $(n, m)$ via teoria da decisão, onde $n$ é o tamanho amostral e $m$ é o grau do polinômio de Bernstein. Além disso, a inclusão de conhecimento a priori através de uma medida de probabilidade pode ser incorporada ao método de estimação. A especificação da priori nesse caso pode ser uma tarefa não trivial, pela complexidade do espaço paramétrico, e seu estudo também se faz necessário. A inclusão de uma medida de incerteza a priori sobre a função de distribuição (desconhecida) permite abordar o problema por uma visão puramente preditivista, justificada pelo Teorema de De Finetti (De Finetti, 1937, 1972, 1974). Por exemplo, na aplicação apresentada na seção 4.4: dado que foram observados $\left(x_{1}, y_{1}\right), \ldots,\left(x_{n}, y_{n}\right)$ e um novo paciente é recrutado com o valor inicial de M-YBOCS igual $x_{n+1}$, é de interesse conhecer a distribuição preditiva $P\left(Y_{n+1} \mid\left(x_{1}, y_{1}\right), \ldots,\left(x_{n}, y_{n}\right), x_{n+1}\right)$ de $Y_{n+1}$, o possível valor de M-YBOCS para esse paciente após o tratamento. 


\section{Apêndice A}

\section{Rotinas computacionais}

Todas as rotinas computacionais apresentadas a seguir foram desenvolvidas no software R (R Development Core Team, 2010).

\section{A.1 Função de distribuição empírica univariada}

Função que retorna o valor da função de distribuição empírica univariada calculada no(s) ponto(s) $\boldsymbol{x}$ com base na amostra contida no vetor $\boldsymbol{X}$.

$1 \mathrm{pEmp}<-$ function $(\mathrm{x}, \mathrm{X})\{\operatorname{ecdf}(\mathrm{X})(\mathrm{x})\}$

\section{A.2 Polinômio de Bernstein univariado}

Função que retorna o(s) valor(es) da função univariada $f$ aproximada pelo polinômio de Bernstein de ordem $m$, calculada nos pontos $\boldsymbol{x}$. A função $f(\boldsymbol{x}, \boldsymbol{X})$ utilizada neste trabalho é a função obtida em A.1. Se o vetor $\boldsymbol{x}$ não contém todos os possíveis valores que $X$ assume, é necessário inserir os limites no vetor lim.

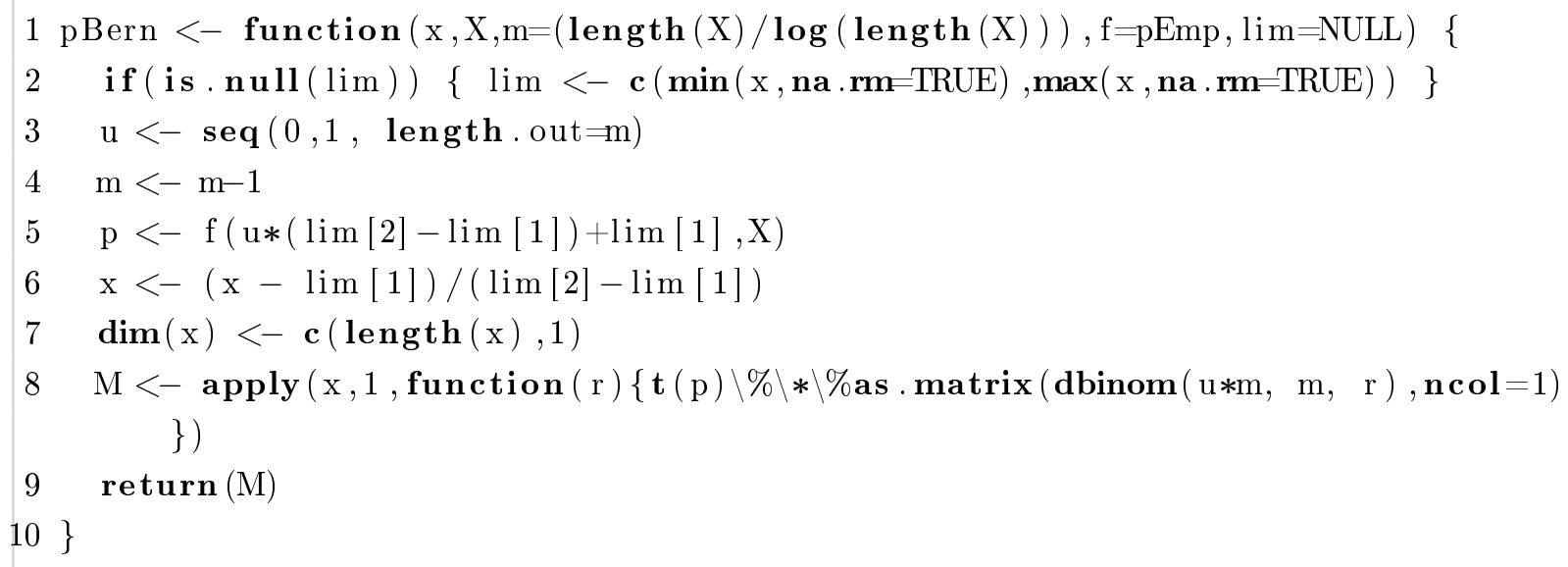




\section{A.3 Função de distribuição empírica bivariada}

Função que retorna a matriz da função de distribuição empírica bivariada calculada no(s) ponto(s) $\boldsymbol{x}$ e $\boldsymbol{y}$ com base na amostra contida nos vetores $\boldsymbol{X}$ e $\boldsymbol{Y}$.

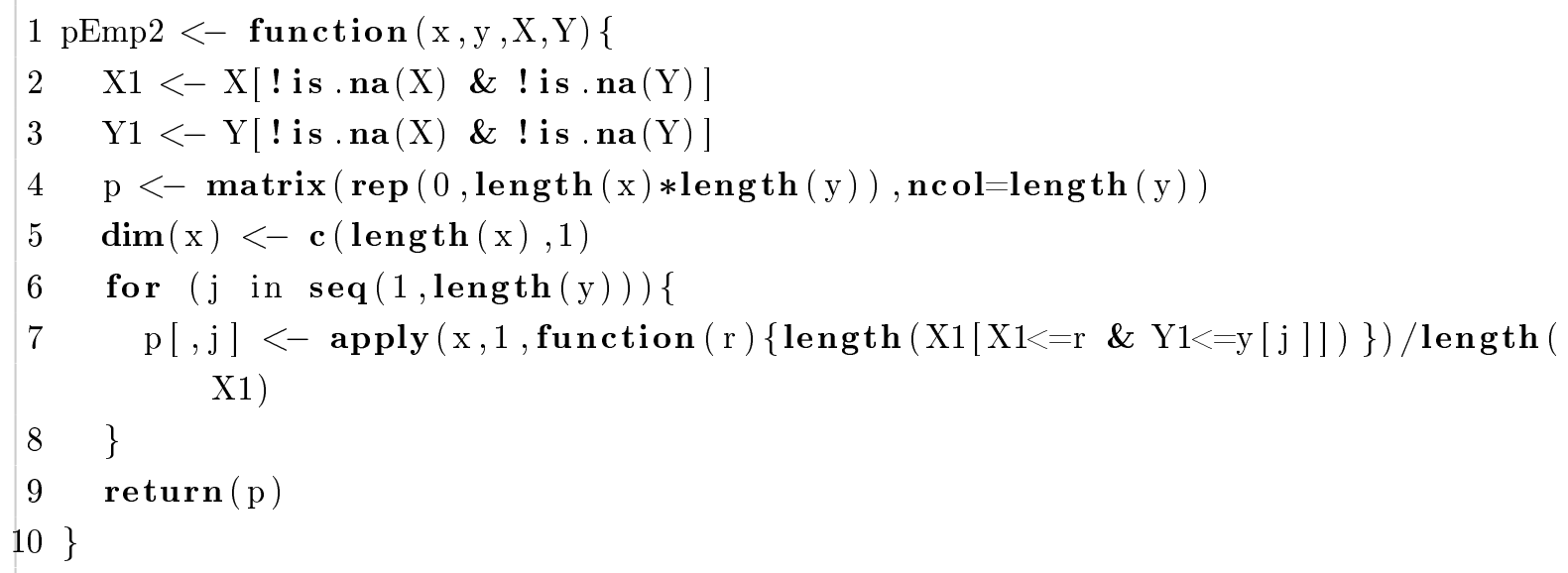

\section{A.4 Função de probabilidade empírica bivariada}

Função que retorna a matriz da função de probabilidade empírica bivariada, calculada em todos os possíveis pares de pontos $(x, y)$ de $\boldsymbol{x}$ e $\boldsymbol{y}$, com base na amostra contida nos vetores $\boldsymbol{X}$ e $\boldsymbol{Y}$.

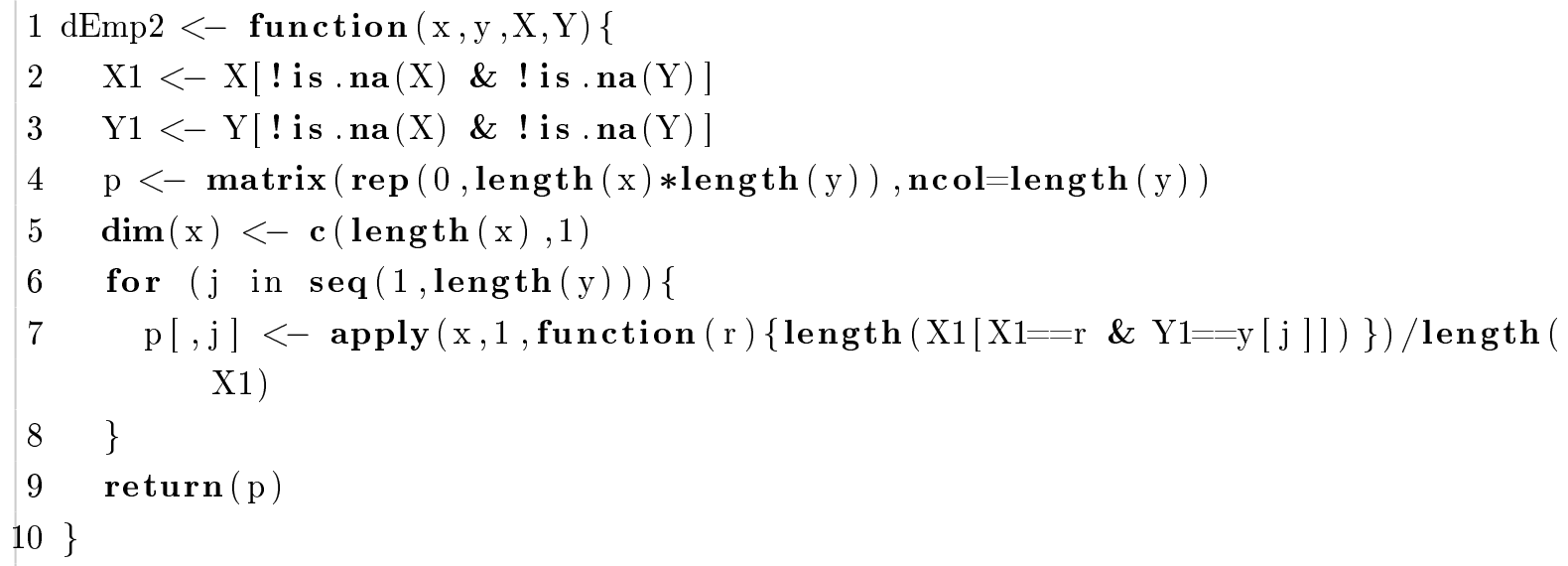

\section{A.5 Função de distribuição bivariada estimada pela dis- tribuição Normal}

Função que retorna a matriz da função de distribuição bivariada estimada pela Normal, calculada em todos os possíveis pares de pontos $(x, y)$ de $\boldsymbol{x}$ e $\boldsymbol{y}$, com base na amostra contida nos vetores $\boldsymbol{X}$ e $\boldsymbol{Y}$. 


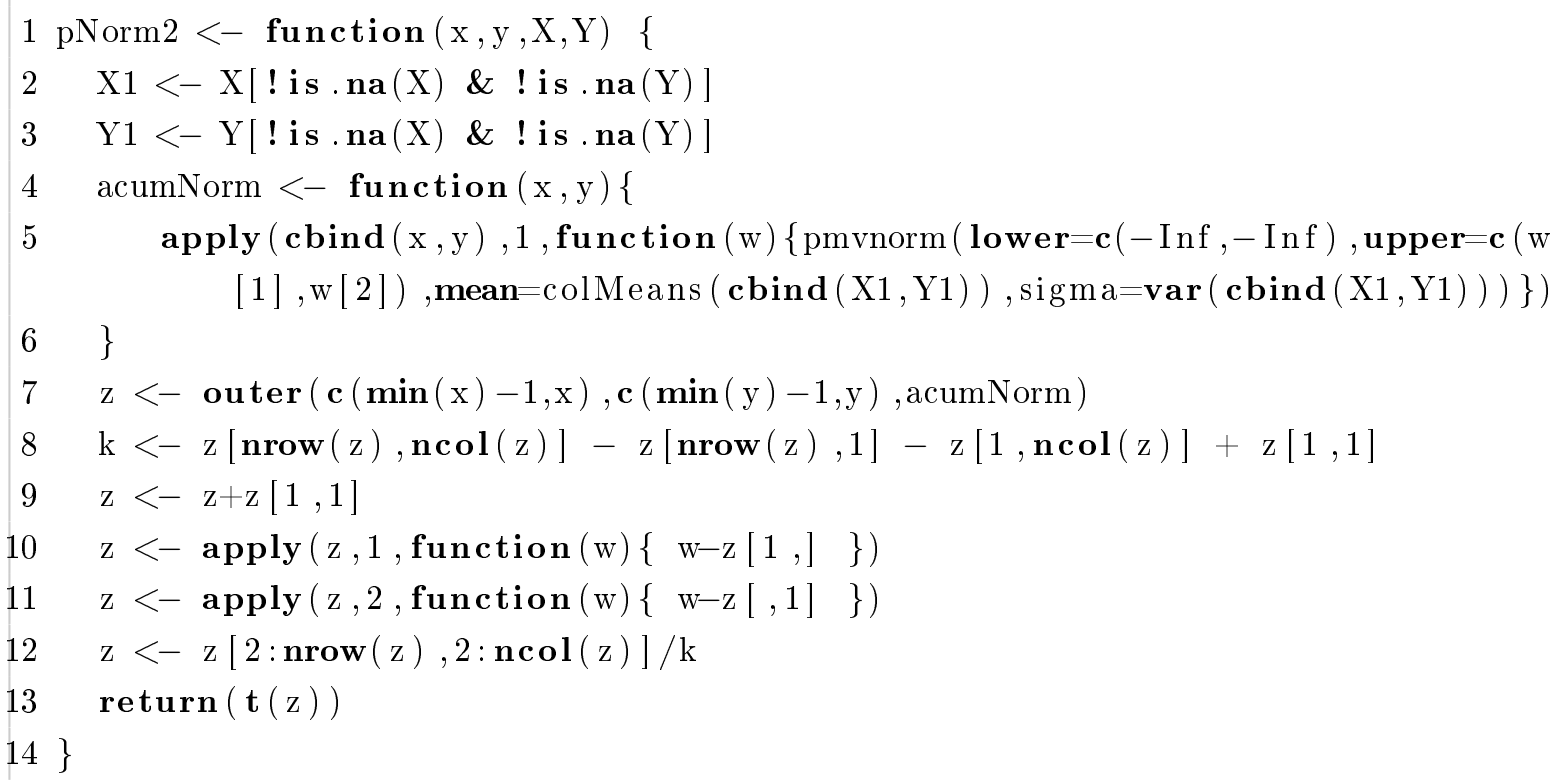

\section{A.6 Polinômio de Bernstein bivariado}

Função que retorna uma matriz do polinômio de Bernstein de ordem $m$ para a aproximar a função bivariada $f$ calculada em todos os possíveis pares de pontos $(x, y)$ de $\boldsymbol{x}$ e $\boldsymbol{y}$. A função $f(\boldsymbol{x}, \boldsymbol{y}, \boldsymbol{X}, \boldsymbol{Y})$ utilizada neste trabalho é a função obtida em A.4. Quando há missing e deseja-se estimar separadamente as marginais e a cópula de Bernstein, o argumento cop deve ser igual a TRUE. Se os vetores $\boldsymbol{x}$ e $\boldsymbol{y}$ não contém todos os possíveis valores que $X$ e $Y$ assumem, é necessário inserir os limites no argumento lim.

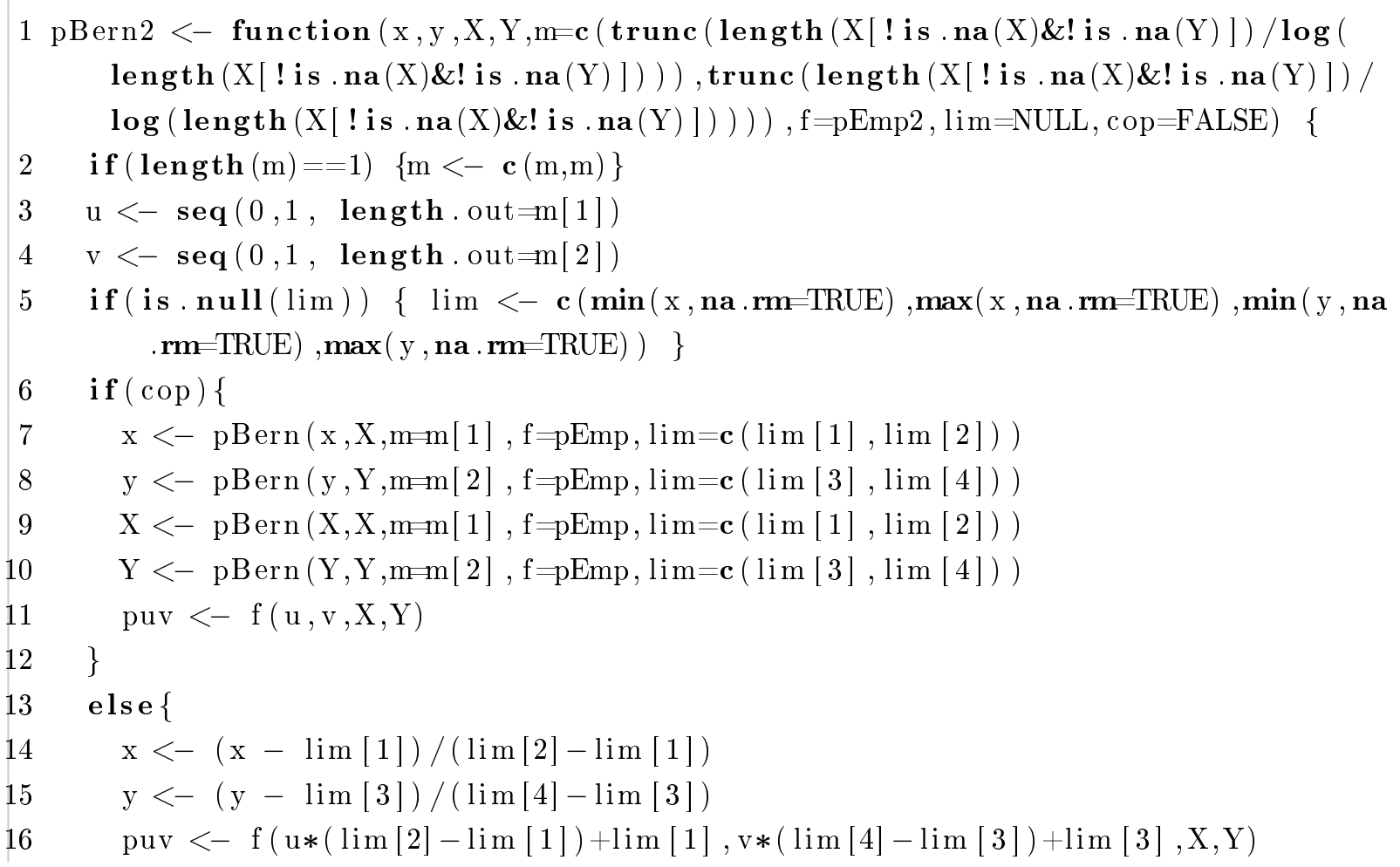


$17\}$

$18 \mathrm{~m}<-\mathrm{m}-1$

$19 \quad \mathrm{xy}<-$ NULL

20 for (i in $1:$ length $(y))\{$

$21 \quad x y<-\operatorname{rbind}(x y, \operatorname{cbind}(x, y[i]))$

$22\}$

$23 \mathrm{M}<-\operatorname{apply}(\mathbf{x y}, 1$, function $(\mathrm{k})\{\mathbf{t}(\mathbf{a s} . \operatorname{matrix}(\mathbf{d b i n o m}(\mathrm{u} * \mathrm{~m}[1], \mathrm{m}[1], \mathrm{k}[1]), \mathbf{n c o l}$

$=1)) \backslash \% \backslash * \backslash \% \operatorname{puv} \backslash \% \backslash * \backslash \% \operatorname{as} . \operatorname{matrix}(\operatorname{dbinom}(\mathrm{v} * \mathrm{~m}[2], \mathrm{m}[2], \mathrm{k}[2]), \mathbf{n c o l}=1)\})$

$24 \operatorname{dim}(\mathrm{M})<-$ c $($ length $(\mathrm{x})$, length $(\mathrm{y}))$

$25 \quad \operatorname{return}(\mathrm{M})$

$26\}$

\section{A.7 Função de probabilidade estimada}

Calcula a matriz correspondente à função de probabilidade bivariada com base na matriz p correspondente à função de distribuição bivariada.

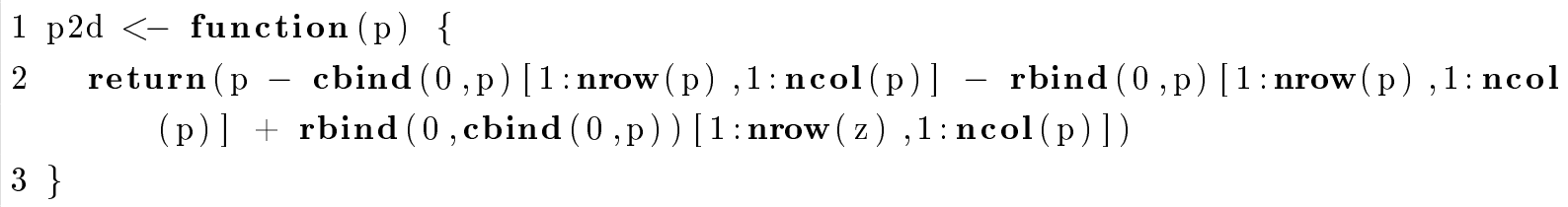

\section{A.8 Distância de Aitchison}

Calcula a distância de Aitchison entre os vetores $\boldsymbol{x}$ e $\boldsymbol{y}$, utilizando a correção $\epsilon$.

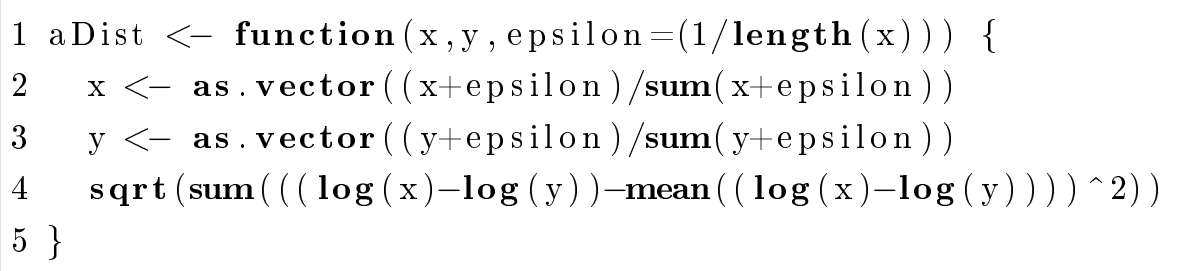

\section{A.9 Gráficos}

Faz gráficos de dispersão para representar as funções de probabilidade e de distribuição discretas, com a intensidade da cor variando conforme a altura da função. A matriz $\boldsymbol{d} \boldsymbol{x} \boldsymbol{y}$ representa a função de distribuição ou de probabilidade dos pares de pontos $\boldsymbol{x}$ e $\boldsymbol{y}$. Os parâmetros $h c$ e $c c$ dizem respeito à cor dos pontos, $p c h$ à forma dos pontos (neste trabalho foram usados $15=$ quadrado e $16=$ círculo) e cex é o tamanho dos pontos, que pode ser uma 
constante, indicando que os pontos terão o mesmo tamanho, ou um vetor ( $\min , \max$ ) indicando que os pontos variam do tamanho min até o max de acordo com o valor de $d x y$. Os demais parâmetros são utilizados como nas rotinas gráficas usuais (para maiores detalhes, use help (par)).

1 Pontos $<-$ function $(\mathrm{x}, \mathrm{y}, \mathrm{dxy}$, add-FALSE, $\mathrm{hc}=0, \mathrm{cc}=1, \mathrm{pch}=16, \mathrm{cex}=1.1$, lab $=\mathbf{c}$ (length

(x), length $(y), 1)$, xlab=NULL, yla $\mathrm{b}=$ NULL, main=NULL, xlim=NULL, ylim=NULL $)\{$

2 dxy $<-$ as . vector $(\mathrm{dxy})$

$3 \quad \mathrm{xy}<-$ NULL

4 for ( $\mathrm{i}$ in $1:$ length $(\mathrm{y}))\{$

$5 \quad \mathrm{xy}<-\operatorname{rbind}(\mathrm{xy}, \operatorname{cbind}(\mathrm{x}, \mathrm{y}[\mathrm{i}]))$

$6\}$

7 if $($ length $($ unique $(\mathrm{dxy}))==1)\{$

8 if (unique $(\mathrm{dxy})==0) \quad\{$

9 print("A matriz de frequências contém apenas zeros!")

10

11 return()

\}

else\{

frc $<-0.5$

cex $<-\operatorname{mean}(\operatorname{cex})$

\}

\}

else \{

$\operatorname{frc}<-0.8 * \mathbf{I}(\mathrm{dxy} !=0) *((\mathrm{dxy}-\min (\mathrm{dxy}[\mathrm{dxy} !=0])) /(\max (\mathrm{dxy}[\mathrm{dxy} !=0])-\boldsymbol{m i n}(\mathrm{dxy}$ $[\mathrm{dxy} !=0])))+0.1 * \mathbf{I}(\mathrm{dxy} !=0)$

cores $<-(1-\mathrm{frc}) * \mathbf{I}(\operatorname{frc} !=0)+1 * \mathbf{I}($ frc $==0)$

\}

if $($ length $(\operatorname{cex})>1)\{$

cex $<-(\max ($ cex, $\mathbf{n a} \cdot \mathbf{r m}=$ TRUE $)-\min (\operatorname{cex}, \mathbf{n a} \cdot \mathbf{r m}=\mathrm{TRUE})) * \mathbf{I}(\mathrm{dxy} !=0) *((\mathrm{dxy}-\mathbf{m i n}($ $\mathrm{dxy}[\mathrm{dxy} !=0])) /(\max (\mathrm{dxy}[\mathrm{dxy} !=0])-\min (\mathrm{dxy}[\mathrm{dxy} !=0])))+\min (\operatorname{cex}, \mathbf{n a} \cdot \mathbf{r m}=$ TRUE $) * \mathbf{I}(\mathrm{dxy} !=0)$

23
\}

i f $($ add $-F A L S E)\{$

plot $(\mathbf{x y}, \mathbf{c o l}=\mathbf{h s v}(\mathrm{hc}, \mathrm{cc} * \mathbf{I}(\mathrm{frc} !=0), \operatorname{cores})$, main $=$ main $, \mathrm{pch}=\mathrm{pch}, \mathrm{cex}=\mathrm{cex}$, $\mathrm{xlab}=\mathrm{xlab}, \quad \mathrm{l} l \mathrm{ab}=\mathrm{ylab}, \mathrm{xlim}=\mathrm{xlim}, \quad \mathrm{lim}=\mathrm{ylim}, \mathrm{lab}=\mathrm{lab}, \quad \mathrm{bg}=$ " transparent")

\}

else \{

points $(\mathbf{x y}, \mathbf{c o l}=\mathbf{h s v}(\mathrm{hc}, \mathrm{cc} * \mathbf{I}(\mathrm{frc} !=0)$, cores $)$, main $=$ main $, \mathrm{pch}=\mathrm{pch}, \mathrm{cex}=\mathrm{cex}$, $\mathrm{xlab}=\mathrm{xlab}, \quad \mathrm{l} l \mathrm{ab}=\mathrm{ylab}, \mathrm{xlim}=\mathrm{xlim}, \quad \mathrm{lim}=\mathrm{ylim}, \mathrm{lab}=\mathrm{lab}, \quad \mathrm{bg}=$ " transparent")

\}

require ( aqfig )

co $<-$ unique $($ cores ) [ unique (cores) $!=1]$ [ order (unique (cores) [unique (cores) !

$=1]$, na. last $=\mathrm{NA}$, decreasing $=\mathrm{TRUE})]$

zl $<-$ unique $(d x y)[\operatorname{unique}(d x y) !=0][\operatorname{order}(\operatorname{unique}(\mathrm{dxy})[\operatorname{unique}(\mathrm{dxy}) !=0]$, na . l as t $=\mathrm{NA})$ ] 


\section{A.10 Distribuição do Exemplo 1}

Funções para a construção da função de distribuição, da função de probabilidade e para simular observações do vetor aleatório $\left(X_{1}, Y_{1}\right)$ descrito no exemplo da seção 4.2.

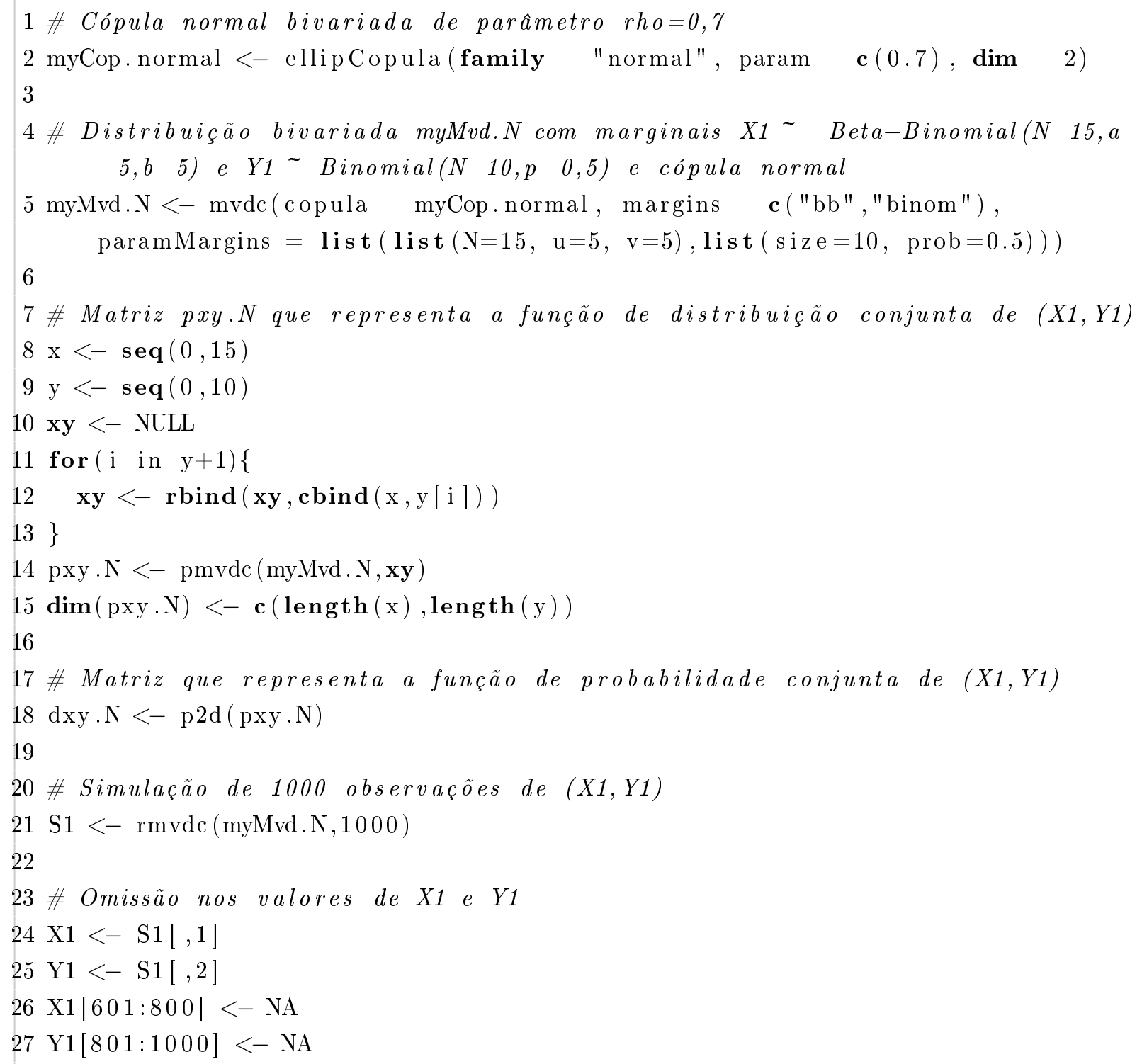

\section{A.11 Distribuição do Exemplo 2}

Funções para a construção da função de distribuição, da função de probabilidade e para simular observações do vetor aleatório $\left(X_{2}, Y_{2}\right)$ descrito no exemplo da seção 4.3. 
1 \# Cópula de Gumbel bivariada de parâmetro theta=2

2 myCop.gumbel <- archmCopula (family = "gumbel", $\mathbf{d i m}=2$, param $=2$ )

3

4 \# Distribuição bivariada myMvd.G com marginais X2 Beta-Binomial $(N=15, a$ $=0,85, b=1,1)$ e $Y^{2} \sim \operatorname{Binomial}(N=10, p=0,6)$ e cópula de Gumbel

5 myMvd.G $<-\operatorname{mvdc}($ copula $=$ myCop.gumbel, margins $=\mathbf{c}(" b b "$, "binom" $)$, paramMargins $=\mathbf{l i s t}(\mathbf{l} \mathbf{i s t}(\mathrm{N}=15, \mathrm{u}=0.85, \mathrm{v}=1.1), \mathbf{l i s t}(\operatorname{size}=10, \operatorname{prob}=0.6)))$

6

7 \# Matriz pxy.N que representa a função de distribuição conjunta de (X2, Y2)

$8 \times<-\operatorname{seq}(0,15)$

$9 \mathrm{y}<-\operatorname{seq}(0,10)$

$10 \mathbf{x y}<-$ NULL

11 for $(i$ in $y+1)\{$

$12 \quad \mathrm{xy}<-\operatorname{rbind}(\mathrm{xy}, \operatorname{cbind}(\mathrm{x}, \mathrm{y}[\mathrm{i}]))$

$13\}$

14 pxy.G $<-\operatorname{pmvdc}(\operatorname{myMvd} . \mathrm{G}, \mathbf{x y})$

$15 \operatorname{dim}(\operatorname{pxy} . G)<-$ c $($ length $(x)$, length $(y))$

16

17 \# Matriz que representa a função de probabilidade conjunta de (X2, Y2)

$18 \mathrm{dxy} \cdot \mathrm{G}<-$ p $2 \mathrm{~d}(\mathrm{pxy} \cdot \mathrm{G})$

19

20 \# Simulação de 1000 observações de (X2, Y2)

$21 \mathrm{~S} 2<-\operatorname{rmvdc}(\operatorname{myMvd} . \mathrm{G}, 1000)$

22

23 \# Omissão nos valores de X2 e Y2

$24 \mathrm{X} 2<-\mathrm{S} 2[, 1]$

$25 \mathrm{Y} 2<-\mathrm{S} 2[, 2]$

$26 \mathrm{X} 2[601: 800]<-\mathrm{NA}$

$27 \mathrm{Y} 2[801: 1000]<-\mathrm{NA}$ 
44 APÊNDICE A 


\section{Referências Bibliográficas}

Aitchison(2003) J. Aitchison. The Statistical Analysis of Compositional Data. Blackburn Press, Caldwell, NJ, USA. ISBN 9781930665781. Citado na pág. 19, 21

Aitchison(2008) J. Aitchison. The single principle of compositional data analysis, continuing fallacies, confusions and misunderstandings and some suggested remedies. Relatório técnico, Universitat de Girona. Departament d'Informàtica i Matemàtica Aplicada. Citado na pág. 20,21

Babu e Chaubey(2006) G. J. Babu e Y. P. Chaubey. Smooth estimation of a distribution and density function on a hypercube using Bernstein polynomials for dependent random vectors. Statistics \&f Probability Letters, 76(9):959-969. Citado na pág. 17, 36

Babu et al.(2002) G. J. Babu, A. J. Canty, e Y. P. Chaubey. Application of Bernstein polynomials for smooth estimation of a distribution and density function. Journal of Statistical Planning and Inference, 105(2):377-392. Citado na pág. 12, 17, 31

Basu e Pereira(1982) D. Basu e C. A. B. Pereira. On the bayesian analysis of categorical data: the problem of nonresponse. Journal of Statistical Planning and Inference, 6(4): 345-362. Citado na pág. 35

Berg(2009) D. Berg. Copula goodness-of-fit testing: an overview and power comparison. The European Journal of Finance, 15(7-8):675-701. Citado na pág. 12

Bouezmarni et al.(2010) T. Bouezmarni, J. V. K. Rombouts, e A. Taamouti. Asymptotic properties of the Bernstein density copula estimator for $\alpha$-mixing data. Journal of Multivariate Analysis, 101(1):1-10. Citado na pág. 12

Branco e Dey(2001) Márcia D. Branco e Dipak K. Dey. A general class of multivariate skew-elliptical distributions. Journal of Multivariate Analysis, 79(1):99-113. ISSN 0047259X. doi: 10.1006/jmva.2000.1960. URL http://www.sciencedirect.com/science/article/ pii/S0047259X00919602. Citado na pág. 2

Cheng(1995) C. Cheng. The Bernstein polynomial estimator of a smooth quantile function. Statistics \& Probability Letters, 24(4):321-330. Citado na pág. 17 
Cheng e Parzen(1997) C. Cheng e E. Parzen. Unified estimators of smooth quantile and quantile density functions. Journal of Statistical Planning and Inference, 59(2):291-307. Citado na pág. 17

De Finetti(1937) B. De Finetti. Breakthroughs in Statistics, volume 1, chapter Foresight: Its logical laws, its subjective sources, páginas 134-174. Springer-Verlag. Traduzido do Francês por H. E. Kiberg e comentado por R. E. Barlow. Citado na pág. 36

De Finetti(1972) B. De Finetti. Probability, Induction and Statistics: The Art of Guessing. John Wiley \& Sons, England. Citado na pág. 7, 36

De Finetti(1974) B. De Finetti. Theory of Probability: A Critical Introductory Treatment. John Wiley \& Sons, England. Citado na pág. 7, 36

DeVore e Lorentz(1993) R. A. DeVore e G. G. Lorentz. Constructive Approximation. Springer, New York. ISBN 3-540-50627-6. Citado na pág. 12

Durrleman et al.(2000a) V. Durrleman, A. Nikeghbali, e T. Roncalli. Copulas approximation and new families. Relatório técnico, Groupe de Recherche Opérationnelle, Crédit Lyonnais. Citado na pág. 12

Durrleman et al.(2000b) V. Durrleman, A. Nikeghbali, e T. Roncalli. Which copula is the right one? Relatório técnico, Groupe de Recherche Opérationnelle, Crédit Lyonnais. Citado na pág. 2, 12, 36

Fisher(1997) N. I. Fisher. Encyclopedia of Statistical Sciences, volume Update Vol 1, chapter Copulas, páginas 159-163. Wiley, New York. Citado na pág. 2, 9

Fossaluza et al.(2009) V. Fossaluza, J. B. Diniz, B. B. Pereira, E. C. Miguel, e C. A. B. Pereira. Sequential allocation to balance prognostic factors in a psychiatric clinical trial. Clinics, 64(6):511-518. Citado na pág. 21

Genest et al.(2009) C. Genest, B. Rémillard, e D. Beaudoin. Goodness-of-fit tests for copulas: A review and a power study. Insurance: Mathematics and Economics, 44(2): 199-213. Citado na pág. 12

Genton e Loperfido(2005) M. G. Genton e N. M. R. Loperfido. Generalized skewelliptical distributions and their quadratic forms. Annals of the Institute of Statistical Mathematics, 57:389-401. ISSN 0020-3157. URL http://dx.doi.org/10.1007/BF02507031. 10.1007/BF02507031. Citado na pág. 2

Ghosal(2001) S. Ghosal. Convergence rates for density estimation with Bernstein polynomials. The Annals of Statistics, 29(5):1264-1280. Citado na pág. 17 
Goodman et al.(1989a) W. K. Goodman, L. H. Price, S. A. Rasmussen, C. Mazure, P. Delgado, G. R. Heninger, e D. S. Charney. The Yale-Brown obsessive compulsive scale: II. Validity. Archives of general psychiatry, 46(11):1012. Citado na pág. 3

Goodman et al.(1989b) W. K. Goodman, L. H. Price, S. A. Rasmussen, C. Mazure, R. L. Fleischmann, C. L. Hill, G. R. Heninger, e D. S. Charney. The Yale-Brown obsessive compulsive scale: I. Development, use, and reliability. Archives of general psychiatry, 46 (11):1006. Citado na pág. 3

Jensen e Nielsen(2007) F. V. Jensen e T. D. Nielsen. Bayesian Networks and Decision Graphs. Springer Verlag, New York. ISBN 0-387-68282-1. Citado na pág. 35

Joe(1997) H. Joe. Multivariate Models and Dependence Concepts. Chapman \& Hall/CRC, London. ISBN 0-412-07331-5. Citado na pág. 2, 9

Kempthorne e Folks(1971) O. Kempthorne e L. Folks. Probability, Statistics, and Data Analysis. Iowa State University Press Ames^ eIowa Iowa. ISBN 0-813-81285-2. Citado na pág. 11

Koralov e Sinai(2007) L. B. Koralov e Y. G. Sinai. Theory of Probability and Random Processes. Springer Verlag. ISBN 978-3-540-25484-3. Citado na pág. 12

Kulpa(1999) T. Kulpa. On approximations of copulas. International Journal of Mathematics and Mathematical Sciences, 22(2):259-269. Citado na pág. 12

Li et al.(1997) X. Li, P. Mikusiński, H. Sherwood, e M.D. Taylor. Distributions with Given Marginals and Moment Problems, chapter On approximation of copulas, páginas 107-116. Kluwer Academic Publishers. ISBN 0-792-34573-8. Citado na pág. 12

Li et al.(1998) X. Li, P. Mikusiński, e M.D. Taylor. Strong approximations of copulas. Journal of Mathematical Analisys and Applications, 255:608-623. ISSN 0161-1712. Citado na pág. 12

Lorentz(1986) G. G. Lorentz. Bernstein Polynomials. Chelsea Pub Co, New York, segunda edição. ISBN 0-8284-0323-6. Citado na pág. 12

Nelsen(2006) R. B. Nelsen. An Introduction to Copulas. Springer Verlag, New York, segunda edição. ISBN 0-387-28659-4. Citado na pág. 2, 8, 9, 10, 14, 18, 24

Paulino et al.(2003) C. D. Paulino, M. A. Turkman, e B. Murteira. Estatística Bayesiana. Fundação Calouste Gulbenkian, Lisboa. Citado na pág. 16

Paulino e Pereira(1992) C. D. M. Paulino e C. A. B. Pereira. Bayesian analysis of categorical data informatively censored. Communications in Statistics-Theory and Methods, 21(9):2689-2705. Citado na pág. 35 
Paulino e Pereira(1995) C. D. M. Paulino e C. A. B. Pereira. Bayesian methods for categorical data under informative general censoring. Biometrika, 82(2):439-446. Citado na pág. 35

Pawlowsky et al.(2007) V G. Pawlowsky, J. J. Egozcue, e R. T. Delgado. Lecture notes on compositional data analysis. Relatório técnico, Universitat de Girona. Citado na pág. 21

Pereira et al.(2011) C. A. B. Pereira, C. B. Silva, e J. B. Diniz. Clínica Psiquiátrica, chapter 147: Estatística em Psiquiatria. Editora Manole. Citado na pág. 3

Perez e Palacín(1987) J. M. Perez e A. F. Palacín. Estimating the quantile function by Bernstein polynomials. Computational Statistics \& Data Analysis, 5(4):391-397. doi: 10.1016/0167-9473(87)90061-2. Citado na pág. 17

Petrone(1999a) S. Petrone. Random Bernstein polynomials. Scandinavian Journal of Statistics, 26(3):373-393. Citado na pág. 17, 31

Petrone(1999b) S. Petrone. Bayesian density estimation using Bernstein polynomials. The Canadian Journal of Statistics, 27(1):105-126. Citado na pág. 17

Petrone e Wasserman(2002) S. Petrone e L. Wasserman. Consistency of Bernstein polynomial posteriors. Journal of the Royal Statistical Society: Series B (Statistical Methodology), 64(1):79-100. ISSN 1467-9868. Citado na pág. 17

R Development Core Team(2010) R Development Core Team. R: A Language and Environment for Statistical Computing. R Foundation for Statistical Computing, Vienna, Austria, 2010. URL http://www.R-project.org/. Citado na pág. 23, 37

Sancetta(2004) A. Sancetta. Nonparametric estimation of multivariate distributions with given marginals. Cambridge Working Papers in Economics. Citado na pág. 12

Sancetta e Satchell(2004a) A. Sancetta e S. E. Satchell. The Bernstein copula and its applications to modeling and approximations of multivariate distributions. Econometric Theory, 20(3):535-562. Citado na pág. 12

Sancetta e Satchell(2004b) A. Sancetta e S. E. Satchell. Bernstein approximations to the copula function and portfolio optimization. Cambridge Working Papers in Economics. Citado na pág. 12

Sen et al.(2010) P. K. Sen, J. M. Singer, e A. C. P. de Lima. From Finite Sample to Asymptotic Methods in Statistics. Cambridge University Press. Citado na pág. 15

Sklar(1959) A. Sklar. Fonctions de répartition à n dimensions et leurs marges. Publ. Inst. Statistique Univ. Paris, 8:229-231. Citado na pág. 2, 9

Taylor(2009) M. D. Taylor. Bernstein polynomials and n-copulas. Arxiv preprint arXiv:0903.1000. Citado na pág. 12 


\title{
Índice Remissivo
}

\author{
Cópula, 9
}

Archimedianas, 10

definição, 9

empírica, 18

gerada por uma distribuição, 9

limites de Fréchet-Hoeffding, 10

produto, 10

Teorema de Sklar, 9

de Gumbel, 23

Normal, 23

Distância de Aitchison, 19

Distribuição Beta-Binomial, 23

Distribuição empírica, 15

Distribuição Normal Multivariada

estimador de máxima verossimilhança, 16

função de densidade de probabilidade, 15

função de distribuição, 15

função de verossimilhança, 16

Exemplos, 23

Dados de psiquiatria, 29

Distribuição bivariada assimétrica, 26

Distribuição bivariada simétrica, 24

Matriz de covariância, 8

Matriz de correlação, 8

Polinômio de Bernstein

convergência uniforme, 12

definição, 12

Pseudo-observações, 18

Teorema de Sklar, 9 